\title{
The $K^{-} p \rightarrow \Sigma^{0} \pi^{0}$ reaction at low energies in a chiral quark model
}

\author{
Xian-Hui Zhong ${ }^{1,4} *$ and Qiang Zhao ${ }^{2,3,4} \dagger$ \\ 1) Department of Physics, Hunan Normal University, Changsha 410081, P.R. China \\ 2) Institute of High Energy Physics, Chinese Academy of Sciences, Beijing 100049, P.R. China \\ 3) Department of Physics, University of Surrey, Guildford, GU2 7XH, United Kingdom and \\ 4) Theoretical Physics Center for Science Facilities, \\ Chinese Academy of Sciences, Beijing 100049, P.R. China
}

\begin{abstract}
A chiral quark-model approach is extended to the study of the $\bar{K} N$ scattering at low energies. The process of $K^{-} p \rightarrow \Sigma^{0} \pi^{0}$ at $P_{K} \lesssim 800 \mathrm{MeV} /$ c (i.e. the center mass energy $W \lesssim 1.7 \mathrm{GeV}$ ) is investigated. This approach is successful in describing the differential cross sections and total cross section with the roles of the low-lying $\Lambda$ resonances in $n=1$ shells clarified. The $\Lambda(1405) S_{01}$ dominates the reactions over the energy region considered here. Around $P_{K} \simeq 400 \mathrm{MeV} / \mathrm{c}$, the $\Lambda(1520) D_{03}$ is responsible for a strong resonant peak in the cross section. The $\Lambda(1670) S_{01}$ has obvious contributions around $P_{K}=750 \mathrm{MeV} / \mathrm{c}$, while the contribution of $\Lambda(1690) D_{03}$ is less important in this energy region. The non-resonant background contributions, i.e. $u$-channel and $t$-channel, also play important roles in the explanation of the angular distributions due to amplitude interferences. The $u$-channel turns out to have significant destructive interferences with the $\Lambda(1405) S_{01}$ at the forward angles. In contrast, the $t$-channel $K^{*}$ exchange has a constructive interference at the forward angles, while suppresses the cross sections slightly at the backward angles. In the $t$ channel, the $K^{*}$-exchange is more dominant over the $\kappa$-exchange. Our analysis suggests that there exist configuration mixings within the $\Lambda(1405) S_{01}$ and $\Lambda(1670) S_{01}$ as admixtures of the $\left[\mathbf{7 0},{ }^{2} \mathbf{1}, 1 / 2\right]$ and $\left[\mathbf{7 0},{ }^{2} \mathbf{8}, 1 / 2\right]$ configurations. The $\Lambda(1405) S_{01}$ is dominated by $\left[\mathbf{7 0},{ }^{2} \mathbf{1}, 1 / 2\right]$, and $\Lambda(1670) S_{01}$ by $\left[\mathbf{7 0},{ }^{2} \mathbf{8}, 1 / 2\right]$. The mixing angle is also determined. The $\Lambda(1520) D_{03}$ and $\Lambda(1690) D_{03}$ are assigned as the $\left[\mathbf{7 0},{ }^{2} \mathbf{1}, 3 / 2\right]$ and $\left[\mathbf{7 0},{ }^{2} \mathbf{8}, 3 / 2\right]$, respectively.
\end{abstract}

PACS numbers: 21.30.Fe, 25.80.Nv, 13.75.Jz, 12.39.Jh

\section{INTRODUCTION}

The reaction $K^{-} p \rightarrow \Sigma^{0} \pi^{0}$ is of particular interest in the study of baryon resonances and $\bar{K} N$ interaction since there are no isospin-1 baryons contributing here and it gives us a rather clean channel to study the $\Lambda$ resonances, such as $\Lambda(1405) S_{01}, \Lambda(1670) S_{01}, \Lambda(1520) D_{03}$ and $\Lambda(1690) D_{03}$.

In the literatures, many experimental 1, 2, 3, 4, 5, 6, 7, 8, 9, 10, 11, 12 and theoretical efforts 13, 14, 15, 16, 17, $18,19,20,21,22,23,24,25,26,27,28,29,30,31,32,33,34,35,36,37,38,39]$ have been devoted to understanding the nature of the low-lying $\Lambda$ resonances. However, their properties still bare a lot of controversies. For example, in the naive quark model the $\Lambda(1405)$ is classified as the lowest $L=1$ orbital excited $q q q$ state as an $S U(3)$ flavor singlet 40, 41, 42]. Meanwhile, it is also proposed to be a dynamically generated resonance emerging from the interaction of the $\bar{K} N$ and $\pi \Sigma$ with a multi-quark structure [16, 17, 18, 19, 20, 21, 22, 23, 24, 25, 26, 27]. Most of those studies are based on the unitary chiral perturbation theory $(\mathrm{U} \chi \mathrm{PT})$. Such a scenario is developed further which proposes that the $\Lambda(1405)$ could be a superposition of two resonances [20, 21, 22, 29, 30, 31, 32]. Similar mechanisms are studied in various processes [35, 36, 37], such as $K^{-} p \rightarrow \pi^{0} \pi^{0} \Sigma^{0}, \pi^{-} p \rightarrow K^{0} \pi \Sigma$ and $p p \rightarrow p K^{+} \Lambda$, as a support of the dynamically generated states. How to clarify these issues and make a contact with experimental observables are still an open question [10, 11, 43, 44, 45].

On the other hand, it is of great importance to understand the excitation of those low-lying $\Lambda$ states in a quark model framework. Quark model somehow provides a guidance for the underlying effective degrees of freedom within hadrons. In order to probe exotic configurations such as multiquarks and hybrids, one should also have a good understanding of where the non-relativistic constituent quark model (NRCQM) breaks down. Particularly in the sector of hyperon states, there are still a lot of ambiguities to be clarified. Apart from the $\Lambda(1405)$, the $\Lambda(1520)$ and $\Lambda(1670)$ are also suggested to be quasibound states of a meson and a baryon, which are dynamically generated resonances based on the $\mathrm{U} \chi \mathrm{PT}[14,27,33]$. While in the quark model these two states are classified as the lowest $L=1$ orbital excited states with $J^{P}=3 / 2^{-}$and $J^{P}=1 / 2^{-}$, respectively. To clarify the nature of those low-lying $\Lambda$ resonances and their internal effective quark degrees of freedom, more theoretical and experimental studies are needed.

\footnotetext{
* E-mail: zhongxh@ihep.ac.cn

† E-mail: zhaoq@ihep.ac.cn
} 
Recently, the higher precision data of the reaction $K^{-} p \rightarrow \Sigma^{0} \pi^{0}$ at eight momentum beams between 514 and $750 \mathrm{MeV} / \mathrm{c}$ were reported [1], which provides us a good opportunity to study the properties of these low-lying $\Lambda$ resonances. In this work, we make an investigation of the $K^{-} p \rightarrow \Sigma^{0} \pi^{0}$ reaction in a chiral quark model. In this model an effective chiral Lagrangian is introduced to account for the quark-pseudoscalar-meson coupling. Since the quark-meson coupling is invariant under the chiral transformation, some of the low-energy properties of QCD are retained. The chiral quark model has been well developed and widely applied to meson photoproduction reactions 46 , 47, 48, 49, 50, 51, 52, 53, 54]. Its recent extension to describe the process of $\pi N$ scattering [55] and investigate the strong decays of charmed hadrons [56, 57] also turns out to be successful and inspiring.

In the literatures the $\bar{K} N$ scattering has been studied using different approaches, such as the $K$-matrix methods 58 , dispersion relations [59, 60], meson-exchange models [61, 62, 63], coupled-channel approaches [13, 19, 20, 23, 24, 25, 30], and quark models 64]. Compared with these models, our model has several obvious features. One is that only a limited number of parameters will appear in the formalism. In particular, only one parameter is need for the resonances to be coupled to the pseudoscalar meson. This distinguishes from hadronic models where each resonance requires one additional coupling constant as a free parameter. The second is that all the resonances can be treated consistently at quark level. Thus, it has predictive powers when exposed to experimental data, and information about the resonance structures and form factors can be extracted.

In the $K^{-} p \rightarrow \Sigma^{0} \pi^{0}$ reaction, for the $s$-channel, the $K^{-}$- and the $\pi^{0}$-mesons can not couple to the same quark in a baryon, which leads to a strong suppression in the $s$-channel amplitudes. As shown in Fig. 4 , the amplitude $M_{2}^{s}$ is suppressed relative to $M_{3}^{s}$ by a factor of $(-1 / 2)^{n}$ with $n$ for the main quantum number of the NRCQM harmonic oscillator potential [46, 47, 48, 49, 50, 51, 52, 53, 54, 55]. In contrast, it is allowed for the $u$-channel that the kaon and pion are coupled to the same quark. Thus, the $u$-channel gives a large background in the cross section, and has significant destructive interferences with $\Lambda(1405) S_{01}$ at the forward angles. The $t$-channel, dominated by the $K^{*}$ exchange, also plays an important role in the reactions. It suppresses the cross section obviously at the backward angles, while enhances it at the forward angles. We also consider the $t$-channel scalar meson exchange, i.e. $\kappa$, but find its contributions are negligibly small.

The $\Lambda(1405)$ governs the reaction in the whole energy region near threshold which is similar to the $S_{11}(1535)$ dominance in $\pi^{-} p \rightarrow \eta n$ [55]. Around $P_{K}=400 \mathrm{MeV} / \mathrm{c}$, the $\Lambda(1520)$ is responsible for the sharp resonant peak in the total cross section. The contributions of $\Lambda(1670)$ turn out to be important at $P_{K} \simeq 750 \mathrm{MeV} / \mathrm{c}$.

The paper is organized as follows. In the subsequent section, the amplitudes of $s$ - and $u$-channels are obtained. Then, amplitudes of $t$-channel are given in Sec. III. The resonance contributions are separated in Sec. IV. We present our calculations and discussions in Sec. V] Finally, a summary is given in Sec. VI]

\section{AMPLITUDES OF THE $s$ - AND $u$-CHANNEL TRANSITIONS}

\section{A. The interactions}

The effective quark-pseudoscalar-meson coupling in the chiral quark model has been discussed in detail in Refs. 53 , 54, 55]. Here, we only outline the main formulae to keep the self-consistence of this work.

The low energy quark-meson interactions are described by the effective Lagrangian [53, 54]

$$
\mathcal{L}=\bar{\psi}\left[\gamma_{\mu}\left(i \partial^{\mu}+V^{\mu}+\gamma_{5} A^{\mu}\right)-m\right] \psi+\cdots
$$

where $V^{\mu}$ and $A^{\mu}$ correspond to vector and axial currents, respectively. They are given by

$$
\begin{aligned}
V^{\mu} & =\frac{1}{2}\left(\xi \partial^{\mu} \xi^{\dagger}+\xi^{\dagger} \partial^{\mu} \xi\right), \\
A^{\mu} & =\frac{1}{2 i}\left(\xi \partial^{\mu} \xi^{\dagger}-\xi^{\dagger} \partial^{\mu} \xi\right),
\end{aligned}
$$

under the chiral transformation $\xi=\exp \left(i \phi_{m} / f_{m}\right)$, where $f_{m}$ is the meson's decay constant. For the $S U(3)$ case, the pseudoscalar-meson octet $\phi_{m}$ can be expressed as

$$
\phi_{m}=\left(\begin{array}{ccc}
\frac{1}{\sqrt{2}} \pi^{0}+\frac{1}{\sqrt{6}} \eta & \pi^{+} & K^{+} \\
\pi^{-} & -\frac{1}{\sqrt{2}} \pi^{0}+\frac{1}{\sqrt{6}} \eta & K^{0} \\
K^{-} & \bar{K}^{0} & -\sqrt{\frac{2}{3} \eta}
\end{array}\right),
$$


and the quark field $\psi$ is given by

$$
\psi=\left(\begin{array}{l}
\psi(u) \\
\psi(d) \\
\psi(s)
\end{array}\right)
$$

At the leading order of the Lagrangian [Eq.(1)], the quark-meson pseudovector coupling is

$$
H_{m}=\sum_{j} \frac{1}{f_{m}} \bar{\psi}_{j} \gamma_{\mu}^{j} \gamma_{5}^{j} \psi_{j} \vec{\tau} \cdot \partial^{\mu} \vec{\phi}_{m}
$$

where $\psi_{j}$ represents the $j$-th quark field in a hadron.

The non-relativistic form of Eq. (5) can be written as [53, 54, 55]

$$
H_{m}^{n r}=\sum_{j}\left\{\frac{\omega_{m}}{E_{f}+M_{f}} \boldsymbol{\sigma}_{j} \cdot \mathbf{P}_{f}+\frac{\omega_{m}}{E_{i}+M_{i}} \boldsymbol{\sigma}_{j} \cdot \mathbf{P}_{i}-\boldsymbol{\sigma}_{j} \cdot \mathbf{q}+\frac{\omega_{m}}{2 \mu_{q}} \boldsymbol{\sigma}_{j} \cdot \mathbf{p}_{j}^{\prime}\right\} I_{j} \varphi_{m},
$$

where $\boldsymbol{\sigma}_{j}$ corresponds to the Pauli spin vector of the $j$-th quark in a hadron, and $\mu_{q}$ is a reduced mass given by $1 / \mu_{q}=1 / m_{j}+1 / m_{j}^{\prime}$, where $m_{j}$ and $m_{j}^{\prime}$ stand for the masses of the $j$-th quark in the initial and final hadrons, respectively. For emitting a meson, we have $\varphi_{m}=\exp \left(-i \mathbf{q} \cdot \mathbf{r}_{j}\right)$, and for absorbing a meson we have $\varphi_{m}=\exp \left(i \mathbf{q} \cdot \mathbf{r}_{j}\right)$. In the above non-relativistic expansions, $\mathbf{p}_{j}^{\prime}\left(=\mathbf{p}_{j}-\frac{m_{j}}{M} \mathbf{P}_{c . m}\right.$. $)$ is the internal momentum for the $j$-th quark in the initial meson rest frame. $\omega_{m}$ and $\mathbf{q}$ are the energy and three-vector momentum of the light meson, respectively. The isospin operator $I_{j}$ in Eq. (6) is expressed as

$$
I_{j}= \begin{cases}a_{j}^{\dagger}(u) a_{j}(s) & \text { for } K^{+}, \\ a_{j}^{\dagger}(s) a_{j}(u) & \text { for } K^{-}, \\ a_{j}^{\dagger}(d) a_{j}(s) & \text { for } K^{0}, \\ a_{j}^{\dagger}(s) a_{j}(d) & \text { for } \bar{K}^{0}, \\ a_{j}^{\dagger}(u) a_{j}(d) & \text { for } \pi^{+}, \\ a_{j}^{\dagger}(d) a_{j}(u) & \text { for } \pi^{-}, \\ \frac{1}{\sqrt{2}}\left[a_{j}^{\dagger}(u) a_{j}(u)-a_{j}^{\dagger}(d) a_{j}(d)\right] & \text { for } \pi^{0},\end{cases}
$$

where $a_{j}^{\dagger}(u, d, s)$ and $a_{j}(u, d, s)$ are the creation and annihilation operators for the $u, d$ and $s$ quarks.

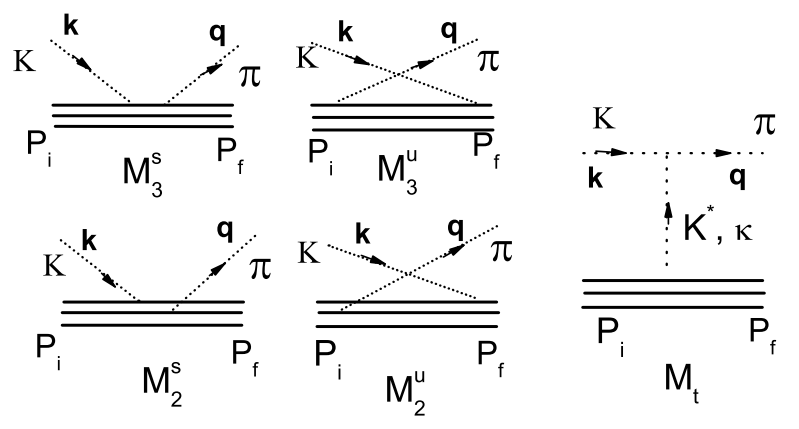

FIG. 1: Transition channels labeled by the Mandelstem variables, i.e. $s, u$, and $t$ - channels. $M_{3}^{s}$ and $M_{3}^{u}\left(M_{2}^{s}, M_{2}^{u}\right)$ correspond to the amplitudes of the $s$ - and $u$-channels with the incoming meson and outgoing meson absorbed and emitted by the same quark (different quarks), respectively. Note that in reaction $K^{-} p \rightarrow \Sigma^{0} \pi^{0}$ the amplitude $M_{3}^{s}$ vanishes. 


\section{B. The $s$-channel amplitudes}

The $s$-channel transition amplitudes as shown in Fig. 1 can be expressed as

$$
\mathcal{M}_{s}=\sum_{j}\left\langle N_{f}\left|H_{\pi}\right| N_{j}\right\rangle\left\langle N_{j}\left|\frac{1}{E_{i}+\omega_{K}-E_{j}} H_{K}\right| N_{i}\right\rangle,
$$

where $\omega_{K}$ is the energy of the incoming $K^{-}$-meson. $H_{K}$ and $H_{\pi}$ are the standard quark-meson couplings at tree level described by Eq.(5). $\left|N_{i}\right\rangle,\left|N_{j}\right\rangle$ and $\left|N_{f}\right\rangle$ stand for the initial, intermediate and final states, respectively, and their corresponding energies are $E_{i}, E_{j}$ and $E_{f}$, which are the eigenvalues of the NRCQM Hamiltonian $\hat{H}$ [40, 65]. Following the procedures developed in Refs. [52, 53, 54, 55], one can then express the $s$-channel amplitudes by operator expansions:

$$
\mathcal{M}_{s}=\sum_{j}\left\langle N_{f}\left|H_{\pi}\right| N_{j}\right\rangle\left\langle N_{j}\left|\sum_{n} \frac{1}{\omega_{K}^{n+1}}\left(\hat{H}-E_{i}\right)^{n} H_{K}\right| N_{i}\right\rangle
$$

where $n$ is the principle harmonic oscillator quantum number. Note that for any operator $\hat{\mathcal{O}}$, one has

$$
\left(\hat{H}-E_{i}\right) \hat{\mathcal{O}}\left|N_{i}\right\rangle=[\hat{H}, \hat{\mathcal{O}}]\left|N_{i}\right\rangle
$$

a systematic expansion of the commutator between the NRCQM Hamiltonian $\hat{H}$ and the vertex coupling $H_{K}$ and $H_{\pi}$ can thus be carried out. Details of this treatment can be found in Refs. [52, 53, 54], but we note that in this study only the spin-independent potential in $\hat{H}$ is considered as a feasible leading order calculation.

Finally, we can obtain the $s$-channel amplitude in the harmonic oscillator basis, which is expressed as [55]

$$
\mathcal{M}^{s}=\sum_{n}\left(\mathcal{M}_{3}^{s}+\mathcal{M}_{2}^{s}\right) e^{-\left(\mathbf{k}^{2}+\mathbf{q}^{2}\right) / 6 \alpha^{2}}
$$

where $\alpha$ is the oscillator strength, and $e^{-\left(\mathbf{k}^{2}+\mathbf{q}^{2}\right) / 6 \alpha^{2}}$ is a form factor in the harmonic oscillator basis. $\mathcal{M}_{3}^{s}\left(\mathcal{M}_{2}^{s}\right)$ corresponds to the amplitudes for the outgoing meson and incoming meson absorbed and emitted by the same quark (different quarks) (see Fig. 1). Because of the isospin selection rule, the $\pi$ and $K^{-}$can not couple to the same quark. Thus, the contribution of $\mathcal{M}_{3}^{s}$ vanishes and only $\mathcal{M}_{2}^{s}$ contributes to the $s$-channel, i.e.

$$
\begin{aligned}
\mathcal{M}_{2}^{s}= & \left\langle N_{f}\right| 6 I_{1}\left\{\boldsymbol{\sigma}_{1} \cdot \mathbf{A}_{\text {out }} \boldsymbol{\sigma}_{3} \cdot \mathbf{A}_{\text {in }} \sum_{n=0} \frac{F_{s}(n)}{n !} \frac{\mathcal{X}^{n}}{(-2)^{n}}+\left[-\boldsymbol{\sigma}_{1} \cdot \mathbf{A}_{\text {out }} \frac{\omega_{K}}{6 \mu_{q}} \boldsymbol{\sigma}_{3} \cdot \mathbf{q}-\frac{\omega_{\pi}}{3 m_{q}} \boldsymbol{\sigma}_{1} \cdot \mathbf{k} \boldsymbol{\sigma}_{3} \cdot \mathbf{A}_{\text {in }}\right.\right. \\
& \left.\left.+\frac{\omega_{\pi}}{m_{q}} \frac{\omega_{K}}{2 \mu_{q}} \frac{\alpha^{2}}{3} \boldsymbol{\sigma}_{1} \cdot \boldsymbol{\sigma}_{3}\right] \times \sum_{n=1} \frac{F_{s}(n)}{(n-1) !} \frac{\mathcal{X}^{n-1}}{(-2)^{n}}+\frac{\omega_{\pi}}{3 m_{q}} \frac{\omega_{K}}{6 \mu_{q}} \boldsymbol{\sigma}_{1} \cdot \mathbf{q} \boldsymbol{\sigma}_{3} \cdot \mathbf{k} \sum_{n=2} \frac{F_{s}(n)}{(n-2) !} \frac{\mathcal{X}^{n-2}}{(-2)^{n}}\right\}\left|N_{i}\right\rangle
\end{aligned}
$$

with

$$
\begin{aligned}
\mathbf{A}_{\text {in }} & =-\left(1+\omega_{K} \mathcal{K}_{i}-\frac{\omega_{K}}{6 \mu_{q}}\right) \mathbf{k}, \\
\mathbf{A}_{\text {out }} & =-\left(1+\omega_{\pi} \mathcal{K}_{f}-\frac{\omega_{\pi}}{3 m_{q}}\right) \mathbf{q},
\end{aligned}
$$

where $\mathcal{K}_{i}=1 /\left(E_{i}+M_{i}\right), \mathcal{K}_{f}=1 /\left(E_{f}+M_{f}\right)$ and $m_{q}$ is the light quark mass. In Eq. (12), the subscriptions of the spin operator $\boldsymbol{\sigma}$ denote that it either operates on quark 3 or quark 1 . The $\mathcal{X}$ is defined as $\mathcal{X} \equiv \frac{\mathbf{k} \cdot \mathbf{q}}{3 \alpha^{2}}$, and the factor $F_{s}(n)$ is given by expanding the energy propagator in Eq. (8) which leads to

$$
F_{s}(n)=\frac{M_{n}}{P_{i} \cdot k-n M_{n} \omega_{h}},
$$

where $M_{n}$ denotes the mass of the excited state in the $n$-th shell, while $\omega_{h}$ is the typical energy of the harmonic oscillator; $P_{i}$ and $k$ are the four momenta of the initial state nucleon and incoming $K^{-}$meson in the c.m. system. The $F_{s}(n)$ has clear physical meaning that recovers the hadronic level propagators. We will come back to this in the next section. 
The above transition amplitude can be written coherently in terms of a number of $g$-factors, which will allow us to relate the quark-level amplitudes to those at hadronic level

$$
\begin{aligned}
\mathcal{M}_{s}= & \left\{g_{s 2} \mathbf{A}_{\text {out }} \cdot \mathbf{A}_{\text {in }} \sum_{n=0}(-2)^{-n} \frac{F_{s}(n)}{n !} \mathcal{X}^{n}+g_{s 2}\left(-\frac{\omega_{K}}{6 \mu_{q}} \mathbf{A}_{\text {out }} \cdot \mathbf{q}-\frac{\omega_{\pi}}{3 m_{q}} \mathbf{A}_{\text {in }} \cdot \mathbf{k}+\frac{\omega_{\pi}}{m_{q}} \frac{\omega_{K}}{2 \mu_{q}} \frac{\alpha^{2}}{3}\right)\right. \\
& \times \sum_{n=1}(-2)^{-n} \frac{F_{s}(n)}{(n-1) !} \mathcal{X}^{n-1}+g_{s 2} \frac{\omega_{\pi} \omega_{K}}{18 m_{q} \mu_{q}} \mathbf{k} \cdot \mathbf{q} \sum_{n=2} \frac{F_{s}(n)}{(n-2) !}(-2)^{-n} \mathcal{X}^{n-2} \\
& +g_{v 2} i \boldsymbol{\sigma} \cdot\left(\mathbf{A}_{\text {out }} \times \mathbf{A}_{\text {in }}\right) \sum_{n=0}(-2)^{-n} \frac{F_{s}(n)}{n !} \mathcal{X}^{n}+g_{v 2} \frac{\omega_{\pi} \omega_{K}}{18 m_{q} \mu_{q}} i \boldsymbol{\sigma} \cdot(\mathbf{q} \times \mathbf{k}) \\
& \left.\times \sum_{n=2}(-2)^{-n} \frac{F_{s}(n)}{(n-2) !} \mathcal{X}^{n-2}\right\} e^{-\left(\mathbf{k}^{2}+\mathbf{q}^{2}\right) / 6 \alpha^{2}},
\end{aligned}
$$

where the $g$-factors, $g_{s 2}$ and $g_{v 2}$, in the $s$-channel are defined as

$$
\begin{aligned}
g_{s 2} & \equiv\left\langle N_{f}\left|\sum_{i \neq j} I_{i}^{\pi} I_{j}^{K} \boldsymbol{\sigma}_{i} \cdot \boldsymbol{\sigma}_{j}\right| N_{i}\right\rangle / 3, \\
g_{v 2} & \equiv\left\langle N_{f}\left|\sum_{i \neq j} I_{i}^{\pi} I_{j}^{K}\left(\boldsymbol{\sigma}_{i} \times \boldsymbol{\sigma}_{j}\right)_{z}\right| N_{i}\right\rangle / 2,
\end{aligned}
$$

which can be derived from the quark model in the $S U(6) \otimes O(3)$ limit.

\section{The $u$-channel amplitudes}

The $u$-channel transition amplitudes (see Fig. 1) are given by

$$
\mathcal{M}_{u}=\sum_{j}\left\langle N_{f}\left|H_{K} \frac{1}{E_{i}-\omega_{\pi}-E_{j}}\right| N_{j}\right\rangle\left\langle N_{j}\left|H_{\pi}\right| N_{i}\right\rangle,
$$

Following the same procedure in the amplitude

$$
\begin{aligned}
\mathcal{M}_{3}^{u}= & -\left\langle N_{f}\right| 3 I_{3}^{K} I_{3}^{\pi}\left\{\boldsymbol{\sigma}_{3} \cdot \mathbf{B}_{\text {in }} \boldsymbol{\sigma}_{3} \cdot \mathbf{B}_{\text {out }} \sum_{n=0} F_{u}(n) \frac{1}{n !} \mathcal{X}^{n}+\left[-\boldsymbol{\sigma}_{3} \cdot \mathbf{B}_{\text {in }} \frac{\omega_{\pi}}{3 m_{q}} \boldsymbol{\sigma}_{3} \cdot \mathbf{k}-\frac{\omega_{K}}{6 \mu_{q}} \boldsymbol{\sigma}_{3} \cdot \mathbf{q} \boldsymbol{\sigma}_{3} \cdot \mathbf{B}_{\text {out }}+\frac{\omega_{\pi}}{m_{q}} \frac{\omega_{K}}{2 \mu_{q}} \frac{\alpha^{2}}{3}\right]\right. \\
& \left.\times \sum_{n=1} F_{u}(n) \frac{\mathcal{X}^{n-1}}{(n-1) !}+\frac{\omega_{\pi}}{3 m_{q}} \frac{\omega_{K}}{6 \mu_{q}} \boldsymbol{\sigma}_{3} \cdot \mathbf{k} \boldsymbol{\sigma}_{3} \cdot \mathbf{q} \sum_{n=2} F_{u}(n) \frac{\mathcal{X}^{n-2}}{(n-2) !}\right\}\left|N_{i}\right\rangle .
\end{aligned}
$$

While the outgoing and incoming mesons couple to two different quarks, the transition amplitude is given by

$$
\begin{aligned}
\mathcal{M}_{2}^{u}= & -\left\langle N_{f}\right| 6 I_{1}^{K} I_{3}^{\pi}\left\{\boldsymbol{\sigma}_{1} \cdot \mathbf{B}_{\text {in }} \boldsymbol{\sigma}_{3} \cdot \mathbf{B}_{\text {out }} \sum_{n=0} \frac{F_{u}(n)}{n !} \frac{\mathcal{X}^{n}}{(-2)^{n}}+\left[-\boldsymbol{\sigma}_{1} \cdot \mathbf{B}_{\text {in }} \frac{\omega_{\pi}}{3 m_{q}} \boldsymbol{\sigma}_{3} \cdot \mathbf{k}-\frac{\omega_{K}}{6 \mu_{q}} \boldsymbol{\sigma}_{1} \cdot \mathbf{q} \boldsymbol{\sigma}_{3} \cdot \mathbf{B}_{\text {out }}\right.\right. \\
& \left.\left.+\frac{\omega_{\pi}}{m_{q}} \frac{\omega_{K}}{2 \mu_{q}} \frac{\alpha^{2}}{3} \boldsymbol{\sigma}_{1} \cdot \boldsymbol{\sigma}_{3}\right] \sum_{n=1} \frac{F_{u}(n)}{(n-1) !} \frac{\mathcal{X}^{n-1}}{(-2)^{n}}+\frac{\omega_{\pi}}{3 m_{q}} \frac{\omega_{K}}{6 \mu_{q}} \boldsymbol{\sigma}_{1} \cdot \mathbf{k} \boldsymbol{\sigma}_{3} \cdot \mathbf{q} \sum_{n=2} \frac{F_{u}(n)}{(n-2) !} \frac{\mathcal{X}^{n-2}}{(-2)^{n}}\right\}\left|N_{i}\right\rangle .
\end{aligned}
$$

In the above equations, we have defined

$$
\begin{aligned}
& \mathbf{B}_{\text {in }} \equiv-\omega_{K}\left(\mathcal{K}_{f}+\mathcal{K}_{j}-\frac{1}{6 \mu_{q}}\right) \mathbf{q}-\left(1+\omega_{K} \mathcal{K}_{j}\right) \mathbf{k} \\
& \mathbf{B}_{\text {out }} \equiv-\omega_{\pi}\left(\mathcal{K}_{i}+\mathcal{K}_{j}-\frac{1}{3 m_{q}}\right) \mathbf{k}-\left(1+\omega_{\pi} \mathcal{K}_{j}\right) \mathbf{q}
\end{aligned}
$$

where $\mathcal{K}_{j}=1 /\left(E_{j}+M_{j}\right)$.

In Eqs.(20) and (21), the factor $F_{u}(n)$ is written as

$$
F_{u}(n)=\frac{M_{n}}{P_{i} \cdot q+n M_{n} \omega_{h}}
$$


where $q$ is the four momentum of the outgoing $\pi$ meson in the c.m. system.

The total amplitude for the $u$-channel is expressed as

$$
\begin{aligned}
\mathcal{M}_{u}= & -\left\{\mathbf{B}_{\text {in }} \cdot \mathbf{B}_{\text {out }} \sum_{n=0}\left[g_{s 1}^{u}+(-2)^{-n} g_{s 2}^{u}\right] \frac{F_{u}(n)}{n !} \mathcal{X}^{n}+\left(-\frac{\omega_{\pi}}{3 m_{q}} \mathbf{B}_{\text {in }} \cdot \mathbf{k}-\frac{\omega_{K}}{3 m_{q}} \mathbf{B}_{\text {out }} \cdot \mathbf{q}+\frac{\omega_{K}}{2 \mu_{q}} \frac{\omega_{\pi}}{m_{q}} \frac{\alpha^{2}}{3}\right)\right. \\
& \times \sum_{n=1}\left[g_{s 1}^{u}+(-2)^{-n} g_{s 2}^{u}\right] \frac{F_{u}(n)}{(n-1) !} \mathcal{X}^{n-1}+\frac{\omega_{\pi} \omega_{K}}{18 m_{q} \mu_{q}} \mathbf{k} \cdot \mathbf{q} \sum_{n=2} \frac{F_{u}(n)}{(n-2) !}\left[g_{s 1}^{u}+(-2)^{-n} g_{s 2}^{u}\right] \mathcal{X}^{n-2} \\
& +i \boldsymbol{\sigma} \cdot\left(\mathbf{B}_{\text {in }} \times \mathbf{B}_{\text {out }}\right) \sum_{n=0}\left[g_{v 1}^{u}+(-2)^{-n} g_{v 2}^{u}\right] \frac{F_{u}(n)}{n !} \mathcal{X}^{n}-\frac{\omega_{\pi} \omega_{K}}{18 m_{q} \mu_{q}} i \boldsymbol{\sigma} \cdot(\mathbf{q} \times \mathbf{k}) \sum_{n=2}\left[g_{v 1}^{u}+(-2)^{-n} g_{v 2}^{u}\right] \\
& \left.\times \frac{F_{u}(n)}{(n-2) !} \mathcal{X}^{n-2}+i \boldsymbol{\sigma} \cdot\left[-\frac{\omega_{\pi}}{3 m_{q}}\left(\mathbf{B}_{\text {in }} \times \mathbf{k}\right)-\frac{\omega_{K}}{6 \mu_{q}}\left(\mathbf{q} \times \mathbf{B}_{\text {out }}\right)\right] \sum_{n=1}\left[g_{v 1}^{u}+(-2)^{-n} g_{v 2}^{u}\right] \mathcal{X}^{n-1} \frac{F_{u}(n)}{(n-1) !}\right\} \\
& \times e^{-\left(\mathbf{k}^{2}+\mathbf{q}^{2}\right) / 6 \alpha^{2}},
\end{aligned}
$$

where the $g$ factors in the $u$-channel are determined by

$$
\begin{aligned}
g_{s 1}^{u} & \equiv\left\langle N_{f}\left|\sum_{j} I_{j}^{K} I_{j}^{\pi}\right| N_{i}\right\rangle, \\
g_{s 2}^{u} & \equiv\left\langle N_{f}\left|\sum_{i \neq j} I_{i}^{K} I_{j}^{\pi} \boldsymbol{\sigma}_{i} \cdot \boldsymbol{\sigma}_{j}\right| N_{i}\right\rangle / 3, \\
g_{v 1}^{u} & \equiv\left\langle N_{f}\left|\sum_{j} I_{j}^{K} I_{j}^{\pi} \sigma_{j}^{z}\right| N_{i}\right\rangle, \\
g_{v 2}^{u} & \equiv\left\langle N_{f}\left|\sum_{i \neq j} I_{i}^{K} I_{j}^{\pi}\left(\boldsymbol{\sigma}_{i} \times \boldsymbol{\sigma}_{j}\right)_{z}\right| N_{i}\right\rangle / 2 .
\end{aligned}
$$

The numerical values of these factors can be derived in the $S U(6) \otimes O(3)$ symmetry limit.

The first term in Eqs. (12), (20) and (21) comes from the correlation between the c.m. motion of the $K^{-}$meson transition operator and the c.m. motion of $\pi$-meson transition operator; the second and the third terms are the correlation among the internal and the c.m. motions of the $K^{-}$and $\pi$ transition operators, and their contributions begin with the $n \geq 1$ exited states in the harmonic oscillator basis. The last two terms in these equations correspond to the correlation of the internal motions between the $K^{-}$and $\pi$ transition operators, and their contributions begin with either $n \geq 1$ or $n \geq 2$ exited states.

\section{AMPLITUDES OF THE $t$-CHANNEL TRANSITIONS}

\section{A. The interactions}

The light meson exchange in the $t$-channel at low energies will generally have larger contributions than the heavy ones. In $K^{-} p \rightarrow \Sigma^{0} \pi^{0}$, we consider the $t$-channel vector meson $K^{*}(892)$ and scalar meson $\kappa(800)$ exchanges which are found dominantly coupled to $K \pi[66]$.

For the $K^{*} K \pi$ and $\kappa K \pi$ couplings, we introduce the following effective interactions

$$
\begin{aligned}
H_{K^{*} K \pi} & =i G_{v}\left\{\left[\left(\partial_{\mu} \bar{K}\right) K^{*}-\bar{K}^{*}\left(\partial_{\mu} K\right)\right] \vec{\tau} \cdot \vec{\pi}-\left[\bar{K} K^{*}-\bar{K}^{*} K\right] \vec{\tau} \cdot\left(\partial_{\mu} \vec{\pi}\right)\right\}, \\
H_{\kappa K \pi} & =\frac{g_{\kappa K \pi}}{2 m_{\pi}} \partial_{\mu} K \partial^{\mu} \pi \kappa,
\end{aligned}
$$

where $G_{v}$ and $g_{\kappa K \pi}$ are the coupling constants to be determined by experimental data [66].

Similar to the quark-pseudoscalar-meson coupling, we introduce the $K^{*} N N$ and $\kappa N N$ couplings at quark level by effective $K^{*} q q$ and $\kappa q q$ Lagrangians:

$$
\begin{aligned}
H_{K^{*} q q} & =\bar{\psi}_{j}\left(a \gamma^{\nu}+\frac{i b \sigma^{\nu \lambda} q_{\lambda}}{2 m_{q}}\right) K_{\nu}^{*} \psi_{j}, \\
H_{\kappa q q} & =g_{\kappa q q} \bar{\psi}_{j} \psi_{j} \kappa
\end{aligned}
$$

where the constants $a, b$ and $g_{\kappa q q}$ are the vector, tensor and scalar coupling constants, which are treated as free parameters in this work. 


\section{B. The amplitudes}

For the vector meson $K^{*}$-exchange, the amplitude of $t$-channel can be written as

$$
\mathcal{M}_{t}^{V}=G_{v}\left(q^{\mu}+k^{\mu}\right) G_{\mu \nu} \sum_{j} \bar{\psi}_{j}\left(a \gamma^{\nu}+\frac{i b \sigma^{\nu \lambda} q_{\lambda}}{2 m_{q}}\right) \phi_{\nu}^{m} \psi_{j}
$$

where $q^{\mu}, k^{\mu}$ are the four momenta of the $\pi^{0}$ and $K^{-}$mesons, respectively. In (34), the propagator $G_{\mu \nu}$ is defined by

$$
G_{\mu \nu}=\left(-g_{\mu \nu}+\frac{Q_{\mu} Q_{\nu}}{t}\right) /\left(t-M_{K^{*}}^{2}\right)
$$

where $t \equiv Q^{2}$. The Feynman diagram is shown in Fig. 1]

The $t$-channel amplitude in the quark model is given by

$$
\mathcal{M}_{t}^{V}=\mathcal{O}_{V}^{t} \frac{1}{t-M_{K^{*}}^{2}} e^{-(\mathbf{q}-\mathbf{k})^{2} / 6 \alpha^{2}},
$$

where $e^{-(\mathbf{q}-\mathbf{k})^{2} / 6 \alpha^{2}}$ is a quark model form factor, $M_{K^{*}}$ is the mass vector meson $K^{*}$, and the amplitude $\mathcal{O}_{V}^{t}$ is given by

$$
\mathcal{O}_{V}^{t}=G_{v} a\left[g_{t}^{s}\left(\mathcal{H}_{0}+\mathcal{H}_{1} \mathbf{q} \cdot \mathbf{k}\right)+g_{t}^{v} \mathcal{H}_{2} i \boldsymbol{\sigma} \cdot(\mathbf{q} \times \mathbf{k})\right]+\text { tensor term }
$$

in Eq. (37) we have defined

$$
\begin{aligned}
\mathcal{H}_{0} & \equiv E_{0}-\left[E_{0} K_{i s}+\left(\mathcal{K}_{i}+\frac{1}{6 \mu_{q}}\right)(1+\mathcal{D})\right] k^{2}+\left[E_{0} \mathcal{K}_{f q}-\left(\mathcal{K}_{f}-\frac{1}{6 \mu_{q}}\right)(1-\mathcal{T})\right] q^{2} \\
\mathcal{H}_{1} & \equiv E_{0}\left[\mathcal{K}_{i} \mathcal{K}_{f}-\left(\mathcal{K}_{f q}-\mathcal{K}_{i s}\right)\right]-\left(\mathcal{K}_{i}+\mathcal{K}_{f}\right)-\left(\mathcal{K}_{i}-\mathcal{K}_{f}\right) \mathcal{T}-\frac{1}{3 \mu_{q}} \mathcal{T} \\
\mathcal{H}_{2} & \equiv E_{0}\left[\mathcal{K}_{f} \mathcal{K}_{i}-\left(\mathcal{K}_{f q}-\mathcal{K}_{i s}\right)\right]-\left(\mathcal{K}_{i}+\mathcal{K}_{f}\right)-\left(\mathcal{K}_{i}-\mathcal{K}_{f}\right) \mathcal{T}+\frac{1}{3}\left(\frac{1}{m_{q}}-\frac{1}{m_{s}}\right) \mathcal{T}
\end{aligned}
$$

with

$$
\begin{aligned}
\mathcal{K}_{f q} & =\frac{1}{6 m_{q}} \mathcal{K}_{f}, \mathcal{K}_{i s}=\frac{1}{6 m_{s}} \mathcal{K}_{i}, \\
\mathcal{T} & =\frac{m_{\pi}^{2}-m_{K}^{2}}{t} \\
E_{0} & =-\left(\omega_{K}+\omega_{\pi}\right)+\left(\omega_{\pi}-\omega_{K}\right) \mathcal{T} .
\end{aligned}
$$

The $K^{*}$ exchange couplings, i.e. vector and tensor, can in principle be determined by $K^{*}$ meson photoproduction. However, it shows that the present experimental results from JLab and ELSA favor quite differently the tensor coupling values. In $K^{-} p \rightarrow \Sigma^{0} \pi^{0}$, the $K^{*}$ exchange is not a predominant transition mechanism. We hence only consider the $t$-channel vector exchange, but neglect the tensor term for simplicity.

In the Eq.(36), we have defined $g_{t}^{s} \equiv\left\langle N_{f}\left|\sum_{j=1}^{3} I_{j}^{K^{-}}\right| N_{i}\right\rangle$, and $g_{t}^{v} \equiv\left\langle N_{f}\left|\sum_{j=1}^{3} \sigma_{j} I_{j}^{K^{-}}\right| N_{i}\right\rangle$, which can be deduced from the quark model. Their values are listed in Tab. [1.

Similarly, for the scalar meson $\kappa$-exchange, the $t$-channel amplitude in the quark model is written as

$$
\mathcal{M}_{t}^{S}=\mathcal{O}_{S}^{t} \frac{1}{t-m_{\kappa}^{2}} e^{-(\mathbf{q}-\mathbf{k})^{2} / 6 \alpha^{2}}
$$

where $m_{\kappa}$ is the $\kappa$-meson mass, and $\mathcal{O}_{S}^{t}$ is given by

$$
\mathcal{O}_{S}^{t} \simeq \frac{g_{\kappa K \pi} g_{\kappa q q}}{2 m_{\pi}}\left(\omega_{K} \omega_{\pi}-\mathbf{q} \cdot \mathbf{k}\right)\left[g_{t}^{s}\left(\mathcal{A}_{0}+\mathcal{A}_{1} \mathbf{q} \cdot \mathbf{k}\right)+g_{t}^{v} \mathcal{A}_{1} i \boldsymbol{\sigma} \cdot(\mathbf{q} \times \mathbf{k})\right]
$$

with

$$
\begin{aligned}
& \mathcal{A}_{0} \equiv 1+\frac{1}{2 m_{q}} \mathcal{K}_{f} \mathbf{q}^{2}-\frac{1}{2 m_{s}} \mathcal{K}_{i} \mathbf{k}^{2} \\
& \mathcal{A}_{1} \equiv \mathcal{K}_{i} \mathcal{K}_{f}-\frac{1}{2 m_{q}} \mathcal{K}_{f}+\frac{1}{2 m_{s}} \mathcal{K}_{i}
\end{aligned}
$$

In Eq.(45), we have neglected the higher order terms. 


\section{SEPARATION OF THE SINGLE RESONANCE CONTRIBUTIONS}

Note that, so far, we have separated out the amplitudes in terms of the harmonic oscillator principle quantum number $n$, which are the sum of a set of $S U(6)$ multiplets with the same $n$. To see the contributions of individual resonances, we need to further separate out the single-resonance-excitation amplitudes within each $n$ in the $s$-channel. Since the resonances in the $u$-channel contribute virtually and are generally suppressed by the kinematics, we treat them as degenerate to $n$.

Function $F_{s}(n)$ in Eq. (15) can be related to the $s$-channel propagator in the infinitely-narrow-width limit:

$$
F_{s}(n)=\frac{2 M_{n}}{s-\left(M_{i}^{2}+M_{K}^{2}+2 n M_{i} \omega_{h}\right)} \equiv \frac{2 M_{n}}{s-M_{n}^{2}},
$$

where it has been assumed that $M_{n}^{2} \equiv M_{i}^{2}+M_{K}^{2}+2 n M_{i} \omega_{h}$, which is not a bad assumption for the masses of an excited $n$-shell state. $M_{i}$ denotes the initial baryon mass.

Taking into account the width effects of the resonances, the resonance transition amplitudes of the $s$-channel can be generally expressed as [54, 55]

$$
\mathcal{M}_{R}^{s}=\frac{2 M_{R}}{s-M_{R}^{2}+i M_{R} \Gamma_{R}} \mathcal{O}_{R} e^{-\left(\mathbf{k}^{2}+\mathbf{q}^{2}\right) / 6 \alpha^{2}},
$$

and the $u$-channel as

$$
\mathcal{M}_{n}^{u}=-\frac{2 M_{n}}{u-M_{n}^{2}} \mathcal{O}_{n} e^{-\left(\mathbf{k}^{2}+\mathbf{q}^{2}\right) / 6 \alpha^{2}}
$$

In Eqs. (49) and (50), $\mathcal{O}_{R}$ is the separated operators for individual resonances in the $s$-channel, while $\mathcal{O}_{n}$ is the operator for a set of degenerate states with the same $n$. In the $s$-channel of $K^{-} p \rightarrow \Sigma^{0} \pi^{0}$, only the $\Lambda$ resonances are involved. Our effort in the following subsections is to extract $\mathcal{O}_{R}$ for each $s$-channel resonance with $n<2$.

\section{A. $n=0$ shell resonances}

With $n=0$ the $\Lambda$-hyperon is the only state contributing to the $s$-channel, and the amplitude can be written as

$$
\mathcal{M}_{\Lambda}^{s}=\mathcal{O}_{\Lambda} \frac{2 M_{\Lambda}}{s-M_{\Lambda}^{2}} e^{-\left(\mathbf{k}^{2}+\mathbf{q}^{2}\right) / 6 \alpha^{2}},
$$

with

$$
\mathcal{O}_{\Lambda}=g_{s 2} \mathbf{A}_{\text {out }} \cdot \mathbf{A}_{\text {in }}+g_{v 2} i \boldsymbol{\sigma} \cdot\left(\mathbf{A}_{\text {out }} \times \mathbf{A}_{\text {in }}\right),
$$

where $M_{\Lambda}$ is the $\Lambda$-hyperon mass.

\section{B. $n=1$ shell resonances}

Both $S$ - and $D$-wave resonances contribute to the $s$-channel amplitude with $n=1$. Note that the spin-independent amplitude for $D$-waves is proportional to the Legendre function $P_{2}^{0}(\cos \theta)$, and the spin-dependent amplitude is in proportion to $\frac{\partial}{\partial \theta} P_{2}^{0}(\cos \theta)$. Moreover, the $S$-wave amplitude is independent of the scattering angle. Thus, the $S$ - and $D$-wave amplitudes can be separated out easily as follows,

$$
\begin{aligned}
& \mathcal{O}_{S}=-\frac{1}{2} g_{s 2}\left(\left|\mathbf{A}_{\text {out }}\right| \cdot\left|\mathbf{A}_{\text {in }}\right| \frac{|\mathbf{k}||\mathbf{q}|}{9 \alpha^{2}}-\frac{\omega_{K}}{6 \mu_{q}} \mathbf{A}_{\text {out }} \cdot \mathbf{q}-\frac{\omega_{\pi}}{3 m_{q}} \mathbf{A}_{\text {in }} \cdot \mathbf{k}+\frac{\omega_{\pi} \omega_{K}}{2 m_{q} \mu_{q}} \frac{\alpha^{2}}{3}\right), \\
& \mathcal{O}_{D}=-\frac{1}{2} g_{s 2}\left|\mathbf{A}_{\text {out }}\right| \cdot\left|\mathbf{A}_{\text {in }}\right|\left(3 \cos ^{2} \theta-1\right) \frac{|\mathbf{k}||\mathbf{q}|}{9 \alpha^{2}}-\frac{1}{2} g_{v 2} i \boldsymbol{\sigma} \cdot\left(\mathbf{A}_{\text {out }} \times \mathbf{A}_{\text {in }}\right) \frac{\mathbf{k} \cdot \mathbf{q}}{3 \alpha^{2}} .
\end{aligned}
$$

In the NRCQM, the $n=1$ shell contains three different representations, i.e. $\left[\mathbf{7 0},{ }^{2} \mathbf{1}\right],\left[\mathbf{7 0},{ }^{2} \mathbf{8}\right]$ and $\left[\mathbf{7 0},{ }^{4} \mathbf{8}\right]$. The two low-lying $\Lambda$-resonances, $\Lambda(1405) S_{01}$ and $\Lambda(1520) D_{03}$, are classified to be flavor singlet states of $\left[\mathbf{7 0}{ }^{2} \mathbf{1}\right]$, and they 
have no counterparts in the nucleon spectrum. The $\Lambda(1670) S_{01}$ and $\Lambda(1690) D_{03}$ are interpreted as multiplets of $\left[\mathbf{7 0},{ }^{2} \mathbf{8}\right]$, which are octet partners of the nucleon resonances $S_{11}(1535)$ and $D_{13}(1520)$. Usually the $\Lambda(1800) S_{01}$ and $\Lambda(1830) D_{05}$ are classified as multiplets of $\left[\mathbf{7 0},{ }^{4} \mathbf{8}\right]$, among which the $D_{03}$ state has not yet been found in experiment. In the $S U(6) \otimes O(3)$ quark model, the contributions of $\left[\mathbf{7 0},{ }^{4} \mathbf{8}\right]$ are forbidden in $K^{-} p \rightarrow \Sigma^{0} \pi^{0}$ due to the so-called " $\Lambda$-selection rule" [70, 71, 72]. Thus, for the $S$-wave, only the resonances, $\Lambda(1405) S_{01}$ and $\Lambda(1670) S_{01}$, contribute to the reactions, and for the $D$-waves, $\Lambda(1520) D_{03}$ and $\Lambda(1690) D_{03}$.

The separated amplitudes for the $S$ - and $D$-wave can thus be re-written as

$$
\begin{aligned}
\mathcal{O}_{S} & =\left[g_{S_{01}(1405)}+g_{S_{01}(1670)}\right] \mathcal{O}_{S}, \\
\mathcal{O}_{D} & =\left[g_{D_{03}(1520)}+g_{D_{03}(1690)}\right] \mathcal{O}_{D},
\end{aligned}
$$

where the factor $g_{R}\left(R=S_{01}(1405)\right.$, etc) represents the resonance transition strengths in the spin-flavor space, and is determined by the matrix element $\left\langle N_{f}\left|H_{\pi}\right| N_{j}\right\rangle\left\langle N_{j}\left|H_{K}\right| N_{i}\right\rangle$. Their relative strengths can be explicitly determined by the following relations

$$
\begin{aligned}
& \frac{g_{S_{01}(1405)}}{g_{S_{01}(1670)}}=\frac{\left\langle N_{f}\left|I_{3}^{\pi} \boldsymbol{\sigma}_{3}\right| S_{01}(1405)\right\rangle\left\langle S_{01}(1405)\left|I_{3}^{K} \boldsymbol{\sigma}_{3}\right| N_{i}\right\rangle}{\left\langle N_{f}\left|I^{\pi} \boldsymbol{\sigma}_{3}\right| S_{01}(1670)\right\rangle\left\langle S_{01}(1670)\left|I_{3}^{K} \boldsymbol{\sigma}_{3}\right| N_{i}\right\rangle} \\
& \frac{g_{D_{03}(1520)}}{g_{D_{03}(1690)}}=\frac{\left\langle N_{f}\left|I_{3}^{\pi} \boldsymbol{\sigma}_{3}\right| D_{03}(1520)\right\rangle\left\langle D_{03}(1520)\left|I_{3}^{K} \boldsymbol{\sigma}_{3}\right| N_{i}\right\rangle}{\left\langle N_{f}\left|I^{\pi} \boldsymbol{\sigma}_{3}\right| D_{03}(1690)\right\rangle\left\langle D_{03}(1690)\left|I_{3}^{K} \boldsymbol{\sigma}_{3}\right| N_{i}\right\rangle} .
\end{aligned}
$$

On the condition of no configuration mixing among these states, we have $g_{S_{01}(1405)} / g_{S_{01}(1670)}=g_{D_{03}(1520)} / g_{D_{03}(1690)}=$ -3 . However, the admixtures of different configurations usually occur in physical states with the same quantum number due to spin-dependent forces [40, 42]. We shall see in Sec $\nabla$ that configuration mixing may exist between the $S$-wave $S_{01}(1405)$ and $S_{01}(1670)$ in this reaction. By allowing the data to constraint the relative partial strengths, i.e. $g_{S_{01}(1405)} / g_{S_{01}(1670)}$, we can extract the mixing angle as a leading order result.

With the same method, we can separate the amplitudes in $n=2$ shell as well, the detail can be found in our previous work [55]. In this work, the higher resonances (i.e. $n \geq 2$ ) are treated as degenerate since they are less important in the beam momentum region $P_{K} \lesssim 800 \mathrm{MeV} / \mathrm{c}$ where high precision data are available.

TABLE I: Various $\mathrm{g}$ and $g_{R}$ factors defined in this work and extracted in the symmetric quark model.

\begin{tabular}{|c|c|c|c|}
\hline \hline factor & value & factor & value \\
\hline$g_{s 1}^{u}$ & $1 / 2$ & $g_{t}^{s}$ & $\sqrt{2} / 2$ \\
$g_{s 2}^{u}$ & $2 / 3$ & $g_{t}^{v}$ & $-\sqrt{2} / 6$ \\
$g_{v 1}^{u}$ & $-1 / 6$ & $g_{S_{01}(1405)}$ & $3 / 2$ \\
$g_{v 2}^{u}$ & -1 & $g_{S_{01}(1670)}$ & $-1 / 2$ \\
$g_{s 2}$ & $2 / 3$ & $g_{D_{03}(1520)}$ & $3 / 2$ \\
$g_{v 2}$ & 1 & $g_{D_{03}(1690)}$ & $-1 / 2$ \\
\hline
\end{tabular}

\section{CALCULATION AND ANALYSIS}

\section{A. Parameters}

With the transition amplitudes derived from the previous section, the differential cross section can be calculated,

$$
\frac{d \boldsymbol{\sigma}}{d \Omega}=\frac{\left(E_{i}+M_{i}\right)\left(E_{f}+M_{f}\right)}{64 \pi^{2} s} \frac{|\mathbf{q}|}{|\mathbf{k}|} \frac{1}{2} \sum_{\lambda_{i}, \lambda_{f}}\left|\left[\frac{\delta^{2}}{f_{\pi} f_{K}}\left(\mathcal{M}_{s}+\mathcal{M}_{u}\right)+\mathcal{M}_{t}^{V}+\mathcal{M}_{t}^{S}\right]_{\lambda_{f}, \lambda_{i}}\right|^{2}
$$

where $\lambda_{i}= \pm 1 / 2$ and $\lambda_{f}= \pm 1 / 2$ are the helicities of the initial and final state baryons, respectively; $\delta$ is a global parameter accounting for the flavor symmetry breaking effects arising from the quark - meson couplings, and will be determined by experimental data; $f_{\pi}$ and $f_{K}$ are the $\pi$ - and $K$-mesons decay constants, respectively.

To take into account the relativistic effects, we introduce Lorentz boost factors in the spatial part of the amplitudes as done in Refs. [52, 55], i.e.

$$
\mathcal{O}_{i}(\mathbf{k}, \mathbf{q}) \rightarrow \gamma_{k} \gamma_{q} \mathcal{O}_{i}\left(\mathbf{k} \gamma_{k}, \mathbf{q} \gamma_{q}\right)
$$


where $\gamma_{k}=M_{i} / E_{i}$ and $\gamma_{q}=M_{f} / E_{f}$.

We also introduce an energy-dependent width for the resonances in order to take into account the off-mass-shell effects in the reaction [49, 52, 54]:

$$
\Gamma(\mathbf{q})=\Gamma_{R} \frac{\sqrt{s}}{M_{R}} \sum_{i} x_{i}\left(\frac{\left|\mathbf{q}_{i}\right|}{\left|\mathbf{q}_{i}^{R}\right|}\right)^{2 l+1} \frac{D\left(\mathbf{q}_{i}\right)}{D\left(\mathbf{q}_{i}^{R}\right)}
$$

where $\left|\mathbf{q}_{i}^{R}\right|=\left(\left(M_{R}^{2}-M_{b}^{2}+m_{i}^{2}\right) / 4 M_{R}^{2}-m_{i}^{2}\right)^{1 / 2}$, and $\left|\mathbf{q}_{i}\right|=\left(\left(s-M_{b}^{2}+m_{i}^{2}\right) / 4 s-m_{i}^{2}\right)^{1 / 2} ; x_{i}$ is the branching ratio of the resonance decaying into a meson with mass $m_{i}$ and a baryon with mass $M_{b}$, and $\Gamma_{R}$ is the total decay width of the $s$-channel resonance with mass $M_{R} . D(\mathbf{q})=e^{-\mathbf{q}^{2} / 3 \alpha^{2}}$ is a fission barrier function.

In the calculation, the universal value of harmonic oscillator parameter $\alpha=0.4 \mathrm{GeV}$ is adopted. The masses of the $u, d$, and $s$ constituent quarks are set as $m_{u}=m_{d}=330 \mathrm{MeV}$, and $m_{s}=450 \mathrm{MeV}$, respectively. The decay constants for $\pi$, and $K$ are $f_{\pi}=132 \mathrm{MeV}$ and $f_{K}=160 \mathrm{MeV}$, respectively.

Coupling constants in the $t$-channel transitions, i.e. $G_{v}, a, g_{\kappa K \pi}$ and $g_{\kappa q q}$, can be determined by other experimental data. For instance, $G_{v}$ can be determined by $K^{*} \rightarrow K \pi$ [66], while vector coupling a can be extracted from $K^{*}$ photoproduction [48, 68, 69]. As shown by Refs. 68, 69], coupling $a$ has a value of about 3, but with quite significant uncertainties. As $G_{v}$ and $a$ appear simultaneously in the product of $G_{v} a$, we find that $G_{v} a=38$ is a reasonable value for the $K^{*}$ exchange. Note that within the uncertainties of $K^{*} N \Sigma$ coupling, this value can be regarded as reasonable. The value of $g_{\kappa K \pi}$ predicted by QCD sum rules is $g_{\kappa K \pi} \simeq 4$, which is compatible with the value extracted from the data [67]. This implies that the $\kappa q q$ coupling constant is $g_{\kappa q q} \simeq 5$, which also turns to be reasonable.

Parameters in the $s$ - and $u$-channel will be determined by fitting the cross section data. So far, there are 63 datum points of differential cross section at seven momentum beams between 514 and $687 \mathrm{MeV} / \mathrm{c}$ available [1]. By fitting this datum set, we find $\delta \simeq 1.55$ accounting for flavor symmetry breaking effects, and resonance parameters are also determined and listed in the Tab. III From the table, we see that all the resonance parameters roughly agree with the PDG values. The preferred Breit-Wigner mass of the $\Lambda(1405) S_{01}$ is $1420 \mathrm{MeV}$, which is about $10 \mathrm{MeV}$ larger than the upper limit of the PDG suggestion [66]. To fit the total cross section, we find the widths of $\Lambda(1520) D_{03}$ should have a narrower width $\Gamma \simeq 8 \mathrm{MeV}$, which is only half of the PDG value. The fitted mass and width for $\Lambda(1670) S_{01}$ are $M=1697$ and $\Gamma=65 \mathrm{MeV}$, respectively, which are also slightly larger than the PDG suggestions. For the $n=2$ shell we take a degenerate mass and width as $M=1850 \mathrm{MeV}$ and $\Gamma=100 \mathrm{MeV}$ since in the low energy region contributions from the $n=2$ shell are not significant.

TABLE II: Breit-Wigner masses $M_{R}$ (in $\mathrm{MeV}$ ) and widths $\Gamma_{R}$ (in $\mathrm{MeV}$ ) for the resonances in the $s$-channel. States in the $n=2$ shell are treated as degenerate to $n$.

\begin{tabular}{|c|c|c||c|c|}
\hline \hline resonance & $M_{R}$ & $\Gamma_{R}$ & $M_{R}(\mathrm{PDG})$ & $\Gamma_{R}(\mathrm{PDG})$ \\
\hline$S_{01}(1405)$ & 1420 & 48 & $1406 \pm 4$ & $50 \pm 2$ \\
$S_{01}(1670)$ & 1697 & 65 & $1670 \pm 10$ & $25 \sim 50$ \\
$D_{03}(1520)$ & 1520 & 8 & $1520 \pm 1$ & $16 \pm 1$ \\
$D_{03}(1690)$ & 1685 & 63 & $1690 \pm 5$ & $60 \pm 10$ \\
$\mathrm{n}=2$ & 1850 & 100 & & \\
\hline
\end{tabular}

In the $u$-channel, the intermediate states are the nucleon and its resonances. We find that contributions from the $n \geq 1$ shell are negligibly small, and are insensitive to the degenerate masses and widths for these shells. In this work, we take $M_{1}=1650 \mathrm{MeV}\left(M_{2}=1750 \mathrm{MeV}\right), \Gamma_{1}=230 \mathrm{MeV}\left(\Gamma_{2}=300 \mathrm{MeV}\right)$ for the degenerate mass and width of $n=1(n=2)$ shell nucleon resonances, respectively.

The last parameter we consider is the relative strength $g_{S_{01}(1405)} / g_{S_{01}(1670)}$. The data favor a much larger value for $g_{S_{01}(1405)}$ relative to $g_{S_{01}(1670)}$. In another word, a much stronger $S$-wave contribution is needed in the explanation of the experimental data. We thus empirically adjust the relative strength between $S_{01}(1405)$ and $S_{01}(1670)$ by a mixing angle (see Sec $\mathrm{VB}$ ). This could be evidence that the single quark interaction picture fails in the description of the dominant $S$-wave amplitude.

\section{B. Configuration mixing}

In the calculations, we find that the relative strength $g_{S_{01}(1405)} / g_{S_{01}(1670)}$ is crucial for reproducing the angular distributions in the differential cross sections. With no configuration mixing, i.e. $g_{S_{01}(1405)} / g_{S_{01}(1670)}=-3$, the data can not be well explained as shown by the dashed curves in Fig. 2. 


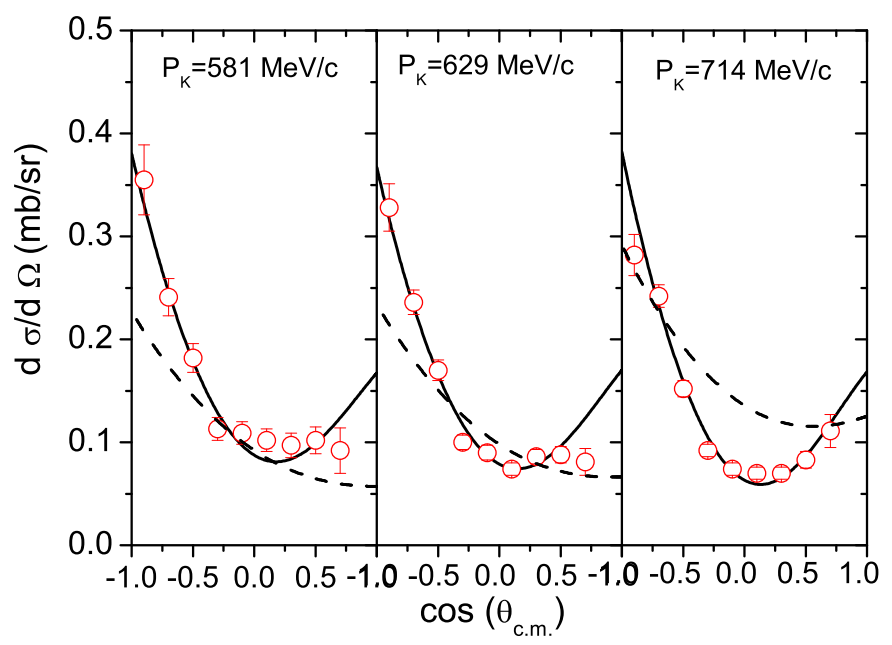

FIG. 2: (Color online) Comparisons of the differential cross sections between with (solid curves) and without configuration mixings (dashed curves) for the $\Lambda(1405)$ and $\Lambda(1670)$, respectively.

As we know, the configuration mixing will bring uncertainties to this value, thus we determine it by fitting the data. When we take $g_{S_{01}(1405)} / g_{S_{01}(1670)} \simeq-9$, the data can be reasonably reproduced (see the solid curves in Fig. 2), which indicates that the configuration mixing in $S_{01}(1405)$ and $S_{01}(1670)$ is needed. If we take the $g_{D_{03}(1520)} / g_{D_{03}(1690)}$ as a free parameter, the fitted value do not change obviously compared with the value of no configuration mixing. Thus, in the calculations, we do not considered the configuration mixing in $D_{03}(1520)$ and $D_{03}(1690)$.

We empirically introduce a mixing angle between $\left[\mathbf{7 0},{ }^{2} \mathbf{1}\right]$ and $\left[\mathbf{7 0},{ }^{2} \mathbf{8}\right]$ within the physical states $S_{01}(1405)$ and $S_{01}(1670)$, i.e.

$$
\begin{gathered}
\left|S_{01}(1405)\right\rangle=\cos (\theta)\left|\mathbf{7 0},{ }^{2} \mathbf{1}\right\rangle-\sin (\theta)\left|\mathbf{7 0},{ }^{2} \mathbf{8}\right\rangle, \\
\left|S_{01}(1670)\right\rangle=\sin (\theta)\left|\mathbf{7 0},{ }^{2} \mathbf{1}\right\rangle+\cos (\theta)\left|\mathbf{7 0},{ }^{2} \mathbf{8}\right\rangle .
\end{gathered}
$$

Inserting these wave functions into Eq.(157), we have

$$
\frac{g_{S_{01}(1405)}}{g_{S_{01}(1670)}}=\frac{[3 \cos (\theta)-\sin (\theta)][\cos (\theta)+\sin (\theta)]}{[3 \sin (\theta)+\cos (\theta)][\sin (\theta)-\cos (\theta)]}
$$

which is a function of the mixing angle $\theta$.

In order to study the relation between the relative coupling strength $g_{S_{01}(1405)} / g_{S_{01}(1670)}$ and mixing angle $\theta$, we define a function of $\theta$ as

$$
f(\theta)=[3 \cos (\theta)-\sin (\theta)][\cos (\theta)+\sin (\theta)]-\frac{g_{S_{01}(1405)}}{g_{S_{01}(1670)}}[3 \sin (\theta)+\cos (\theta)][\sin (\theta)-\cos (\theta)] .
$$

For a given ratio $g_{S_{01}(1405)} / g_{S_{01}(1670)}$, the mixing angle $\theta$ can be determined at $f(\theta)=0$. One can easily check that the ratio $g_{S_{01}(1405)} / g_{S_{01}(1670)}=-3$ leads to $\theta=0^{\circ}$, i.e. no configuration mixing between $\left[\mathbf{7 0},{ }^{2} \mathbf{1}\right]$ and $\left[\mathbf{7 0},{ }^{2} \mathbf{8}\right]$.

With the fitted value $g_{S_{01}(1405)} / g_{S_{01}(1670)}=-9$, the $f(\theta)$ as a function of $\theta$ is shown in Fig. 3. The mixing angle can then be extracted at $f(\theta)=0$. From the figure, we find that two mixing angles, $\theta \simeq 41^{\circ}$ and $165^{\circ}$, satisfy the condition $f(\theta)=0$ with $g_{S_{01}(1405)} / g_{S_{01}(1670)}=-9$.

With $\theta=41^{\circ}$, the admixtures of flavor singlet $\left[\mathbf{7 0},{ }^{2} \mathbf{1}\right]$ and flavor octet $\left[\mathbf{7 0},{ }^{2} \mathbf{8}\right]$ in the $\Lambda(1405)$ amount to $57 \%$ and $43 \%$, respectively. With $\theta=165^{\circ}$, the $\Lambda(1405)$ is dominantly $\left[\mathbf{7 0},{ }^{2} \mathbf{1}\right]$ with a wave function density of $\sim 93 \%$, while admixture of $\left[\mathbf{7 0},{ }^{2} \mathbf{1}\right]$ in $\Lambda(1670)$ is only $\sim 7 \%$. The recent relativistic quark model study suggests that for the $\Lambda(1405)$ the admixtures of singlet $\left[\mathbf{7 0},{ }^{2} \mathbf{1}\right]$ and octet $\left[\mathbf{7 0},{ }^{2} \mathbf{8}\right]$ are $\sim 70 \%$ and $\sim 30 \%$, respectively, and for the $\Lambda(1670)$, the admixture of $\left[\mathbf{7 0},{ }^{2} \mathbf{8}\right]$ is $\sim 62 \%$ and that of $\left[\mathbf{7 0},{ }^{2} \mathbf{1}\right]$ is $\sim 26 \%[42]$, which is compatible with the results with $\theta=41^{\circ}$. It is interesting to note that this feature that the $\Lambda(1405)$ and $\Lambda(1670)$ as mixed states dominated by the singlet and octet, respectively, is also obtained by the coupled channel studies based on $\mathrm{U} \chi \mathrm{PT}[22]$. 
Furthermore, Eq. (64) allows us to investigate the ratios of the couplings to the $\bar{K} N$ and $\pi \Sigma$ channel for the states $S_{01}(1405)$ and $S_{01}(1670)$ with the following relations:

$$
\begin{aligned}
& \frac{g_{S_{01}(1405) \bar{K} N}}{g_{S_{01}(1670) \bar{K} N}}=\frac{\cos (\theta)+\sin (\theta)}{\sin (\theta)-\cos (\theta)}, \\
& \frac{g_{S_{01}(1405) \pi \Sigma}}{g_{S_{01}(1670) \pi \Sigma}}=\frac{3 \cos (\theta)-\sin (\theta)}{3 \sin (\theta)+\cos (\theta)} .
\end{aligned}
$$

If we take the mixing angle $\theta=41^{\circ}$ we have

$$
\left|\frac{g_{S_{01}(1405) \bar{K} N}}{g_{S_{01}(1670) \bar{K} N}}\right| \simeq 14,\left|\frac{g_{S_{01}(1405) \pi \Sigma}}{g_{S_{01}(1670) \pi \Sigma}}\right| \simeq 0.6 .
$$

This solution is in agreement with the UChPT model prediction [24], which also prefers a much stronger coupling of the $S_{01}(1405)$ to the $\bar{K} N$ channel than the $S_{01}(1670)$.

On the other hand, if the mixing angle is taken as $\theta=165^{\circ}$ it gives

$$
\left|\frac{g_{S_{01}(1405) \bar{K} N}}{g_{S_{01}(1670) \bar{K} N}}\right| \simeq 0.58,\left|\frac{g_{S_{01}(1405) \pi \Sigma}}{g_{S_{01}(1670) \pi \Sigma}}\right| \simeq 17 .
$$

In order to determine the mixing angle and the couplings for these two $S_{01}$ states, a coherent study of the photoproduction $\gamma p \rightarrow K^{+} \Lambda(1405)$ and $\gamma p \rightarrow K^{+} \Lambda(1670)$ would be needed.

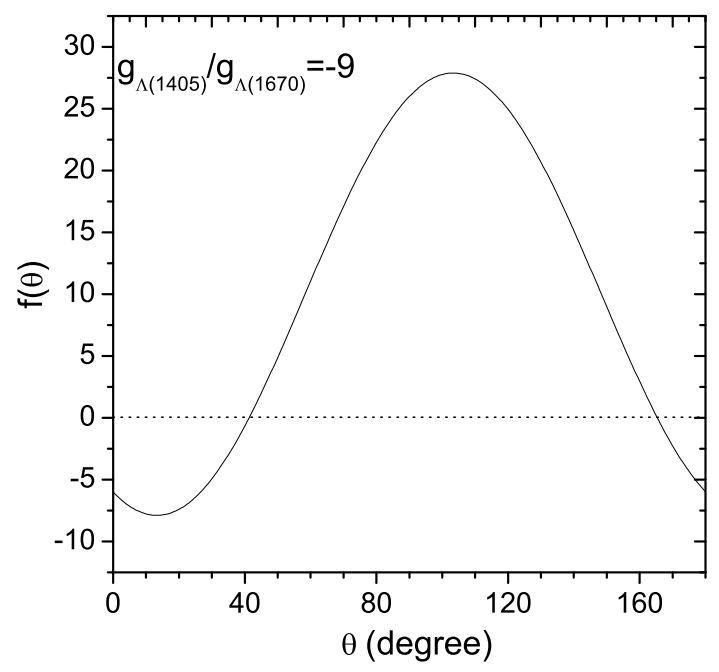

FIG. 3: The evolution of function $f(\theta)$ in terms of the mixing angle $\theta$ is shown. The values of $\theta$ corresponding to $f(\theta)=0$ are the mixing angles for $g_{S_{01}(1405)} / g_{S_{01}(1670)}=-9$, which are found to be $\theta \simeq 41^{\circ}$ and $\theta \simeq 165^{\circ}$.

\section{Differential cross section}

In Fig. 4, the differential cross sections are shown at different center mass energies (beam momenta) from $W=1536$ $\mathrm{MeV}\left(P_{K}=436 \mathrm{MeV} / \mathrm{c}\right)$ to $W=1687 \mathrm{MeV}\left(P_{K}=773 \mathrm{MeV} / \mathrm{c}\right)$. The experimental data [1, 2, 3, 4, 5, 6] are also included for a comparison. As shown by the solid curves, the overall agreement with the experimental data is rather good. However, we also note that the theoretical results seem to slightly underestimate the differential cross sections at forward angles at $W=1536 \sim 1552 \mathrm{MeV}$, which is just around the $\Lambda(1520) D_{03}$ production threshold. Notice 
that the experimental data possess quite large uncertainties, improved measurement in this energy region is needed to clarify the discrepancies.

To the low energy region, i.e. $W=1457 \sim 1532 \mathrm{MeV}$ ( or $P_{K}=200 \sim 425 \mathrm{MeV} / \mathrm{c}$ ), there are no data for the differential cross sections available from experiment. This is the region that the low-lying $\Lambda(1405) S_{01}$ dominates. Therefore, we plot in Fig. 5 the cross sections given by our model in association with exclusive cross sections by single resonance excitations or transitions. We also carry out such a decomposition for the differential cross sections in the region of $W=1569 \sim 1676 \mathrm{MeV}$ in Fig. 6.

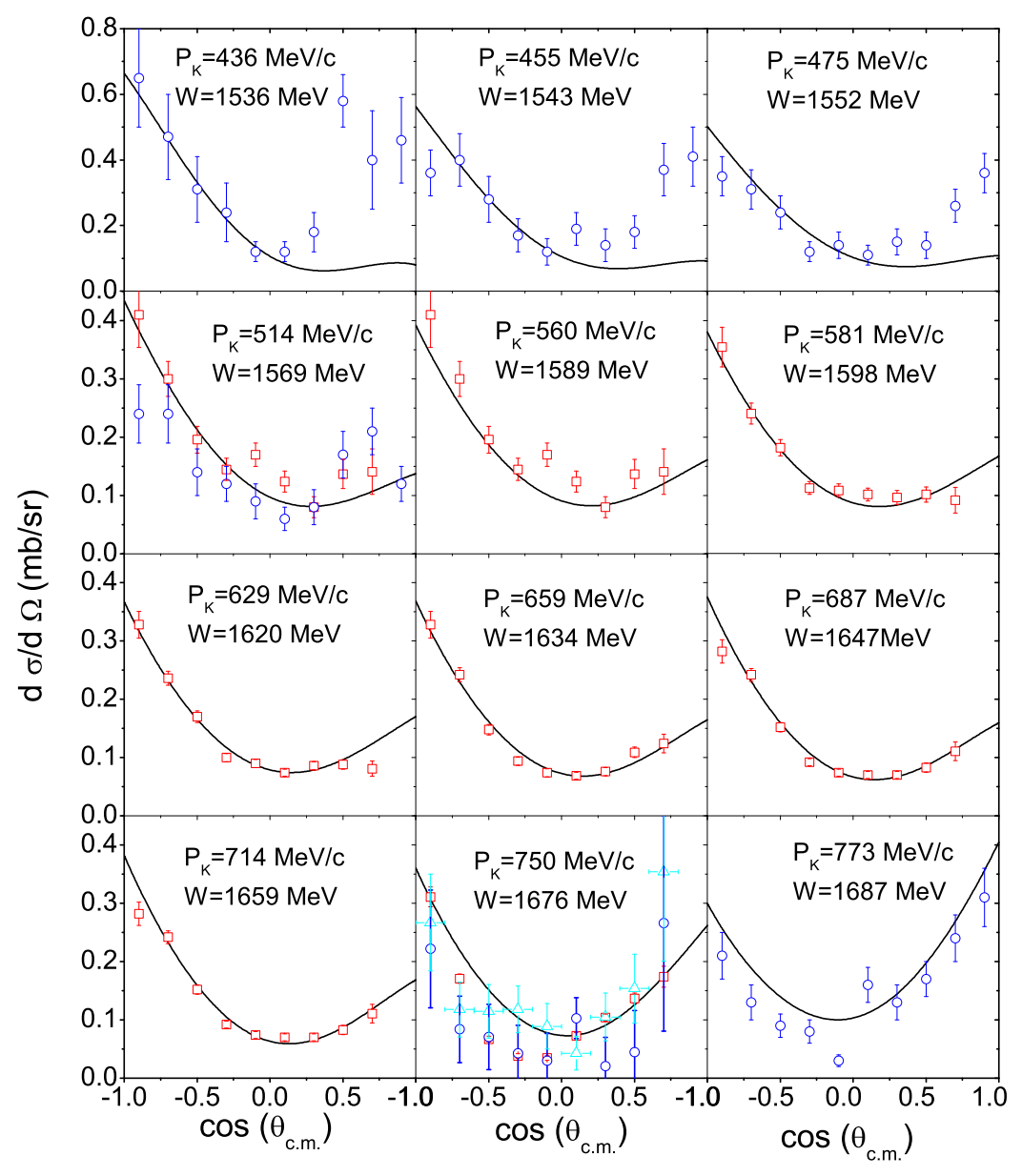

FIG. 4: (Color online) Differential cross sections for $P_{K}=475 \sim 775 \mathrm{MeV} / \mathrm{c}$ (i.e. $W=1536 \sim 1687 \mathrm{MeV}$ ). Data are from [1] (open squares), [4] (open up-triangles) and [2] (open circles).

In Figs. 5 , the solid curves are the full calculations of the model. The thin horizontal lines denote the contributions from the $\Lambda(1405) S_{01}$. Interestingly, the $\Lambda(1405) S_{01}$ appears to be predominant and even larger than the full results. It implies that large cancelations exist between the $\Lambda(1405) S_{01}$ amplitude and other transitions.

The dotted curves in Fig. 5 are contributions from the $u$-channel transition. It presents an enhancement at forward angles though the $u$-channel propagator will generally suppress the forward-angle cross sections. Reason for this enhancement is due to the cancelations occur within the term of $\mathbf{B}_{\text {in }} \cdot \mathbf{B}_{\text {out }}$ at backward angles. Meanwhile, the $u$-channel will provide an important destructive interference with the $\Lambda(1405) S_{01}$, and lower the differential cross sections at the forward direction.

The dash-dotted curves in Fig. 15 represent contributions from the $t$-channel $K^{*}$ exchange, which are also forwardangle enhanced. This contribution deceases with the energies and provides an essentially important interference in 
the amplitudes. As shown in Fig. 6(a) by the dash-dot-dotted curves, its interferences with the rest mechanisms will enhance the forward-angle cross sections, but suppress the backward ones. In contrast, the overall effects from the $t$-channel $\kappa$ exchange are rather small.

At $W=1522 \mathrm{MeV}$ (i.e. $P_{K} \sim 400 \mathrm{MeV} / \mathrm{c}$ ), the contributions from the on-shell $D_{03}(1520)$ can be seen clearly by its interference which significantly changes the shape of the differential cross section. However, in the energies away from its mass, the $D$-wave effects die out quickly.

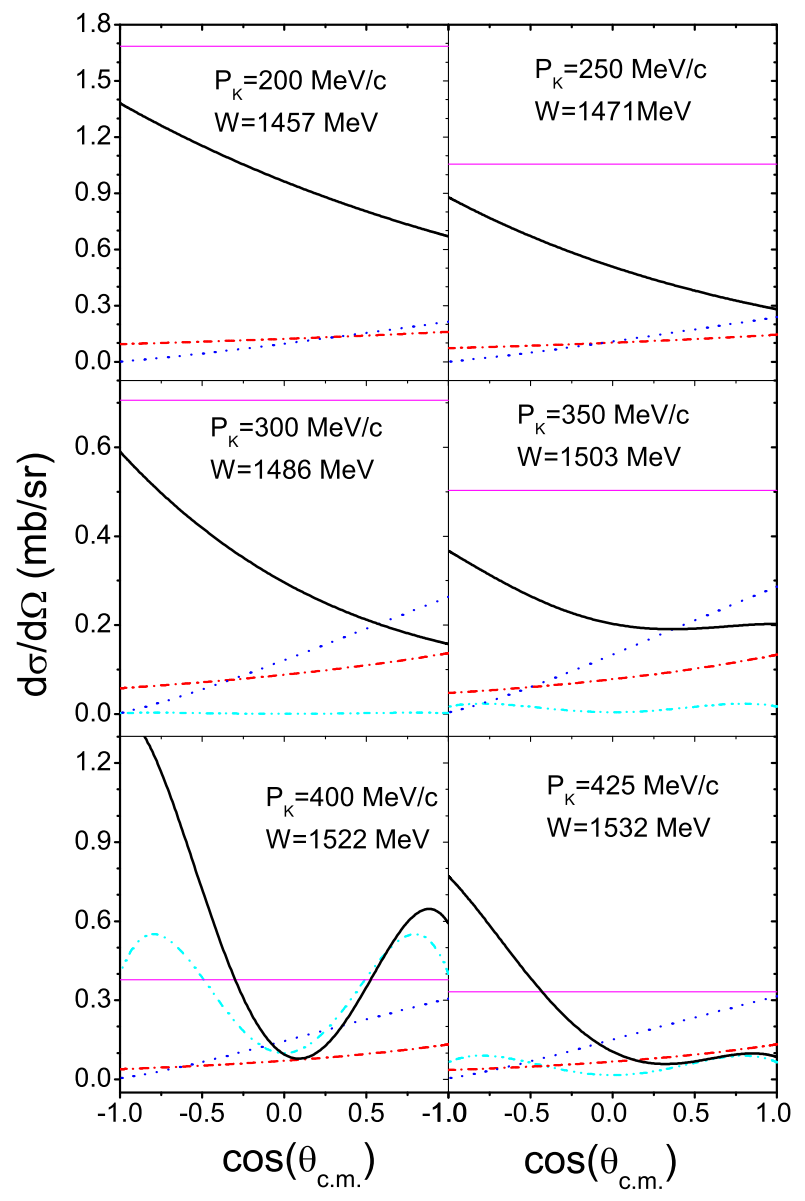

FIG. 5: (Color online) Differential cross sections at six energies in a range of $P_{K}=200 \sim 425 \mathrm{MeV} / \mathrm{c}$ (i.e. $W=1457 \sim 1532$ $\mathrm{MeV}$ ). The bold solid curves are given by the full model calculations. The thin lines, dashed, dash-dotted and dash-dotdotted curves stand for the exclusive cross sections for the $S_{01}(1405), u$-channel, $t$-channel $K^{*}$-exchange, and the $D_{03}(1520)$, respectively.

Further study of the individual transitions are presented in Fig. 6, where in the left panel the cross sections are given by removing one of the transition amplitudes from contributing, while in the right panel cross sections are given by single transitions interfering with the $S$-waves, i.e. $\Lambda(1405) S_{01}$ and $\Lambda(1670) S_{01}$. First in the right panel, the two horizontal lines, thin solid and dash-dotted, are exclusive cross sections for the $\Lambda(1405) S_{01}$ and $\Lambda(1670) S_{01}$, respectively. In the energy region of $W=1569 \sim 1676 \mathrm{MeV}$, the $\Lambda(1405) S_{01}$ is no longer a dominant amplitude though its contribution is still significant. By adding the $\Lambda(1690) D_{03}, \Lambda(1520) D_{03}$, and the $u$-channel to the $S$-waves, their effects are shown by the dashed, dash-dot-dotted, and dotted curves, respectively. It is interesting to see the role played by the $u$-channel, of which the interference contributes to the creation of the backward enhancement. 


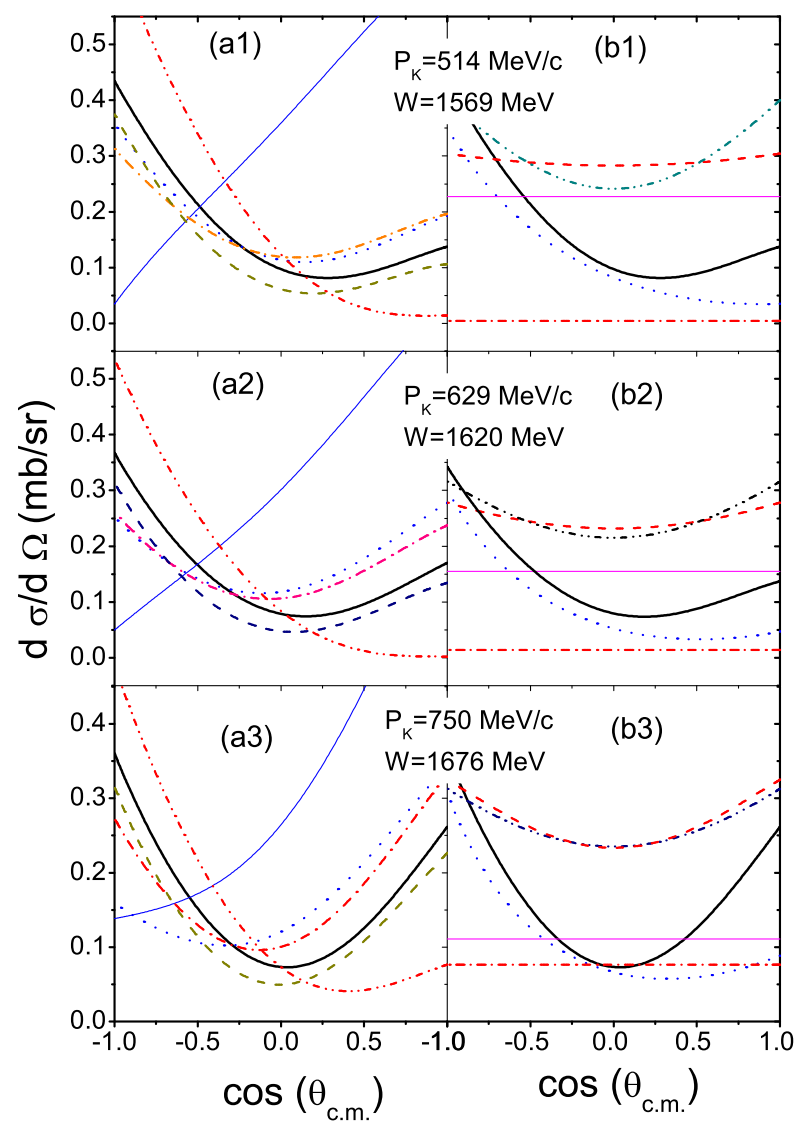

FIG. 6: (Color online) Cross sections of exclusive channels or individual resonances are shown at $P_{K}=514,629$ and 750 $\mathrm{MeV} / \mathrm{c}$, respectively. The bold solid curves are for the full model calculations. In the left panel, i.e. (a1)-(a3), the dashed, dash-dotted, and dash-dot-dotted, dotted and thin solid curves are for the results given by switching off the contributions from the $\Lambda$ pole, $t$-channel $\kappa$-exchange, $t$-channel $K^{*}$-exchange, $\Lambda(1670) S_{01}$, and $\Lambda(1405) S_{01}$, respectively. In the right panel, i.e. (b1)-(b3), the dashed, dash-dot-dotted and dotted curves correspond to the interferences of the $\Lambda(1690) D_{03}, \Lambda(1520) D_{03}$ and $u$-channel with the $S$-wave amplitudes, respectively. The thin solid lines and the dash-dotted lines stand for the exclusive cross sections of the $\Lambda(1405) S_{01}$ and $\Lambda(1670) S_{01}$, respectively.

On the left panel, the thin lines shows the effects without the $\Lambda(1405) S_{01}$, which are strongly forward peaking. Alternatively, this shows how important the $\Lambda(1405) S_{01}$ is in this reaction. The other drastic effects are illustrated by the dash-dot-dotted curves, which are generated by removing the $t$-channel $K^{*}$ exchange. As discussed earlier, it contributes to the forward enhancement and suppresses the backward cross sections. As shown by the dashed, dotted, and dashed-dotted curves, interfering effects from $\Lambda$ pole, $\Lambda(1670) S_{01}$, and $t$-channel $\kappa$ can also be identified. In particular, it shows that the $\kappa$ exchange interferes with the other amplitudes in an opposite behavior in comparison with the $K^{*}$. It suppresses the forward-angle cross sections but enhances the backward ones.

It is interesting to compare this study with $\pi^{-} p \rightarrow \eta n$ [55], where the cross section is also dominated by the $S$-wave near threshold, but the angular distribution is mainly controlled by the $S$ - and $D$-wave interferences. In $K^{-} p \rightarrow \pi^{0} \Sigma^{0}$, we find that the interferences between the $S$-wave and the $u$-channel are more crucial in the energy region $P_{K} \gtrsim 520$ $\mathrm{MeV} / \mathrm{c}$. The $D$-wave interferences become restricted to a relatively narrow energy region due to the narrow width of $\Lambda$ states. It should also be recognized that since only the amplitude $\mathcal{M}_{2}^{s}$ can contribute, the $s$-channel interferences from the $\Lambda$ pole is not as significant as the $u$-channel. 


\section{Total cross section}

The total cross section as a function of the beam momentum is plotted in Fig. 7 to compare with experimental data [1, 2, 3, 4, 5, 6]. To see the contributions of exclusive transitions, their cross sections are also plotted. It shows that our theoretical calculations agree well with the experimental data up to $P_{K}<800 \mathrm{MeV}$.

Towards the low-energy limit, the total cross section exhibits a steep enhancement which is due to the dominant $\Lambda(1405) S_{01}$. The dashed curve shows the exclusive cross section of the $\Lambda(1405) S_{01}$, which is larger than the total cross section of the full calculations. The $u$-channel also turns out to be a major contributor to the cross sections, and is a main background in the whole momentum region. It becomes even larger than the other transitions above $P_{K}>500 \mathrm{MeV}$, and its interference with the $S$-wave amplitudes governs the momentum-dependent behavior of the cross section except for the resonance excitations by the $\Lambda(1520) D_{03}$, which produces a sharp peak in the total cross section. The importance of the $u$-channel contributions are also stressed in the U $\chi \mathrm{PT}$ calculations [15, 32]. It is found there that by switching off the $I=1$ resonances, the results change quite significantly near threshold.

To reproduce this peak, it requires that the $\Lambda(1520) D_{03}$ has a narrow width $\Gamma \simeq 8 \mathrm{MeV}$, which is about a factor 2 smaller than the PDG value. The contributions of the $\Lambda(1670) S_{01}$ are also visible around $P_{K}=0.8 \pm 1 \mathrm{GeV} / \mathrm{c}$. When the beam momentum $P_{K} \gtrsim 800 \mathrm{MeV}$, the model predictions start to become worse, which indicates that the treatment of the resonances of $n=2$ shell as degenerate is no longer applied, and more realistic approach should be introduced. Because of the lack of accurate data in this momentum region, we do not discuss the higher resonances in the $n \geq 2$ shells in this work.

The $t$-channel $K^{*}$ and $\kappa$ exchanges are also shown, and they both decrease with the increase of the beam momentum. Furthermore, the $K^{*}$ exchange is much larger than the $\kappa$ exchange.

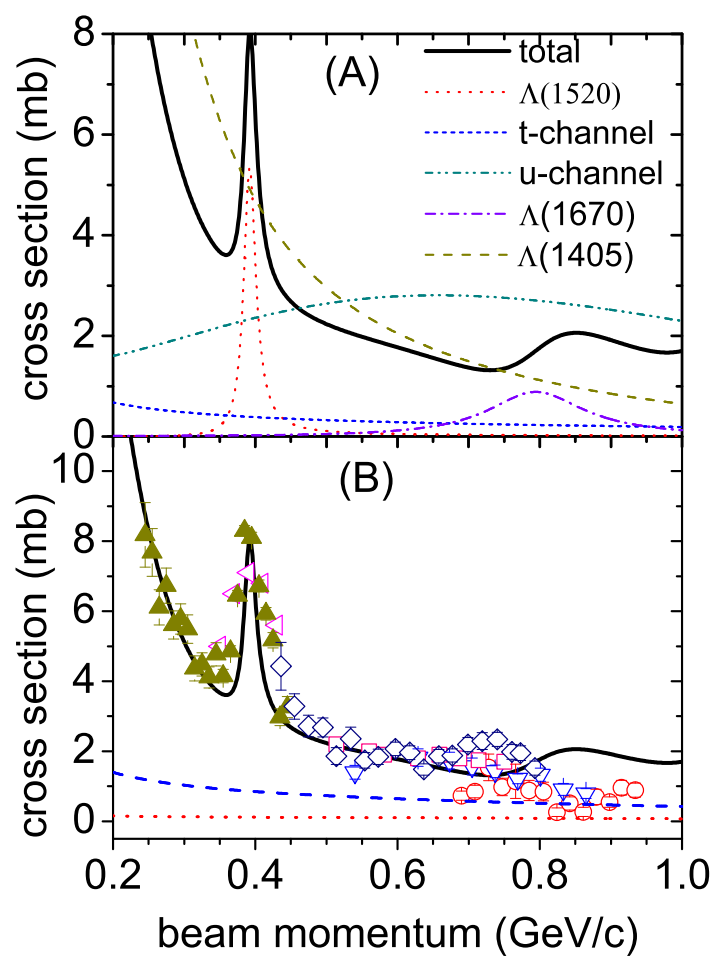

FIG. 7: (Color online) Total cross section as a function of the beam momentum $P_{K}$. The solid curves are the full model calculations. Data are from Refs. [4] (open circles), [6] ( up-triangles), [2] (open diamonds), [5] (left-triangles), [3] (downtriangles), and [1] (squares). In (A), exclusive cross sections for the $\Lambda(1405) S_{01}, \Lambda(1670) S_{01}, \Lambda(1520) D_{03}, t$-channel, and $u$-channel are indicated by different lines, respectively. In (B), the dotted and dashed curves correspond to the exclusive cross sections for the $t$-channel $\kappa$ and $K^{*}$-exchange, respectively. 


\section{SUMMARY AND DISCUSSION}

In this work we have studied the reaction $K^{-} p \rightarrow \Sigma^{0} \pi^{0}$ at low energies within a chiral quark model. With a limited number of parameters, we can describe the differential cross sections and cross sections which are in a good agreement with the data. In the low energy region, i.e., $P_{K}<800 \mathrm{MeV} / \mathrm{c}$, the $n=1$ shell resonances $\Lambda(1405) S_{01}, \Lambda(1520) D_{03}$ and $\Lambda(1670) S_{01}$ are found to play important roles in the reactions, and the $n \geq 2$ shell resonance contributions are negligible small.

The $\Lambda(1405) S_{01}$ is very crucial in the reactions. It is the major contributor of the $S$-wave amplitude in the low-energy region. In particular, in the region of $P_{K} \lesssim 300 \mathrm{MeV} / \mathrm{c}, \Lambda(1405) S_{01}$ dominates the amplitudes, and contributions of the other resonances are nearly invisible in the total cross section. Around $P_{K}=400 \mathrm{MeV} / \mathrm{c}$, the $\Lambda(1520) D_{03}$ is responsible for the strong resonant peak in the total cross section. Around $P_{K}=800 \mathrm{MeV} / \mathrm{c}$, the differential cross sections are sensitive to the $\Lambda(1670) S_{01}$. In this energy region the role of $\Lambda(1690) D_{03}$ is visible, but less important than $\Lambda(1670) S_{01}$.

The non-resonant backgrounds, $u$ - and $t$-channel, also play important roles in the reaction. In the $t$-channel, the $K^{*}$-exchange has larger cross sections than the $\kappa$. It enhances the cross section obviously at the forward angles, and has some destructive interferences at the backward angles. There can be seen a small contribution of the $s$-channel $\Lambda$-pole, which slightly enhances the cross section.

The $u$-channel significantly suppresses the differential cross section at the forward angles, and produces the characteristic backward enhancement. The significant contributions of the $u$-channel agree with the results of $\mathrm{U} \chi \mathrm{PT}[15,32]$. In the quark model framework, the $u$-channel allows transitions that the initial and final state mesons can be coupled to the same quark or different quarks, while the $s$-channel can only occur via transitions that the initial and final state mesons are coupled to different quarks. This explains the importance of the $u$-channel contributions. In comparison with the $\mathrm{U} \chi \mathrm{PT}$, the agreement implies some similarity of the coupling structure at leading order. For instance, the meson-quark couplings in our model can be related to the meson-baryon couplings via current conservation such as the recognition of the Goldberger-Treiman relation [73].

Our analysis suggests that there exist configuration mixings within the $\Lambda(1405) S_{01}$ and $\Lambda(1670) S_{01}$ as admixtures of the $\left[\mathbf{7 0},{ }^{2} \mathbf{1}, 1 / 2\right]$ and $\left[\mathbf{7 0},{ }^{2} \mathbf{8}, 1 / 2\right]$ configurations. The $\Lambda(1405) S_{01}$ is dominated by $\left[\mathbf{7 0},{ }^{2} \mathbf{1}, 1 / 2\right](93 \%$ or $57 \%)$, and $\Lambda(1670) S_{01}$ by $\left[\mathbf{7 0},{ }^{2} \mathbf{8}, 1 / 2\right]$ (93\% or $57 \%$ ), which is in agreement with the U $\chi \mathrm{PT}$ results 22]. The $\Lambda(1520) D_{03}$ and $\Lambda(1690) D_{03}$ are assigned as the $\left[\mathbf{7 0},{ }^{2} \mathbf{1}, 3 / 2\right]$ and $\left[\mathbf{7 0},{ }^{2} \mathbf{8}, 3 / 2\right]$, respectively. This prescription indicates that the $\Lambda(1405) S_{01}, \Lambda(1520) D_{03}$ and $\Lambda(1670) S_{01}$ still possess features of the traditional 3-quark states though they may also have some exotic properties which are not sensitive to the measurement of the cross sections. Experimental measurement of polarization observables may be more selective for exposing their natures, especially for the $\Lambda(1405) S_{01}$. Nevertheless, more accurate differential cross sections in the low beam momentum region, e.g. $P_{K}=200 \sim 500$ $\mathrm{MeV} / \mathrm{c}$, should also be useful.

For higher resonances, we expect more accurate data in the region of $P_{K}=750 \sim 900 \mathrm{MeV} / \mathrm{c}$ can be useful for clarifying their contributions and properties. With such data available, we can then further study the role of $\Lambda(1670) S_{01}, \Lambda(1690) D_{03}$ and the other higher $P$ - and $F$-wave resonances in the $n=2$ shell. The J-PARC facilities, which start to run recently, will provide great opportunities for the study of the hyperon spectrum in theory.

By comparing with approaches at hadronic level, so far we have not yet included the coupled-channel dynamics. It would be interesting and extremely useful to develop a coupled-channel calculation in our framework for baryon resonance excitations in meson-nucleon scattering and meson photoproduction. This would be a natural way of restoring unitarity of the theory, and provide a microscopic description for meson-baryon couplings. Nevertheless, with the coupled-channel effects, one should be able to compare the quark model form factors with those extracted from the hadronic models. We wish to report the progress in the near future.

\section{Acknowledgements}

This work is supported, in part, by the National Natural Science Foundation of China (Grants 10675131 and 10775145), Chinese Academy of Sciences (KJCX3-SYW-N2), and the U.K. EPSRC (Grant No. GR/S99433/01). The authors would like to thank useful discussions with A. Sibirtsev, T.-S.H. Lee, and B.-S. Zou for very useful discussions on relevant issues.

[1] R. Manweiler et al., Phys. Rev. C 77, 015205 (2008).

[2] R. Armenteros et al., Nucl. Phys. B 21, 15 (1970) 
[3] G. W. London et al., Nucl. Phys. B 85, 289 (1975)

[4] D. F. Baxter et al., Nucl. Phys. B 67, 125 (1973).

[5] D. Berley et al., Phys. Rev. D 1, 1996 (1970) [Erratum-ibid. D 3, 2297 (1971)].

[6] T. S. Mast, M. Alston-Garnjost, R. O. Bangerter, A. S. Barbaro-Galtieri, F. T. Solmitz and R. D. Tripp, Phys. Rev. D 11, 3078 (1975).

[7] R. O. Bangerter, M. Alston-Garnjost, A. Barbaro-Galtieri, T. S. Mast, F. T. Solmitz and R. D. Tripp, Phys. Rev. D 23, 1484 (1981).

[8] A. Starostin et al. [Crystal Ball Collaboration], Phys. Rev. C 64, 055205 (2001).

[9] S. Prakhov et al. [Crystall Ball Collaboration], Phys. Rev. C 70, 034605 (2004).

[10] I. Zychor et al., Phys. Lett. B 660, 167 (2008).

[11] I. Zychor, arXiv:0809.3857] [nucl-ex].

[12] M. Niiyama et al., Phys. Rev. C 78, 035202 (2008).

[13] B. Borasoy, U. G. Meissner and R. Nissler, Phys. Rev. C 74, 055201 (2006).

[14] S. Sarkar, E. Oset and M. J. Vicente Vacas, Phys. Rev. C 72, 015206 (2005).

[15] J. A. Oller, Eur. Phys. J. A 28, 63 (2006).

[16] R. H. Dalitz and S. F. Tuan, Phys. Rev. Lett. 2, 425 (1959).

[17] R. H. Dalitz and S. F. Tuan, Annals Phys. 10, 307 (1960)

[18] N. Kaiser, T. Waas and W. Weise, Nucl. Phys. A 612, 297 (1997).

[19] E. Oset and A. Ramos, Nucl. Phys. A 635, 99 (1998).

[20] J. A. Oller and U. G. Meissner, Phys. Lett. B 500, 263 (2001).

[21] V. K. Magas, E. Oset and A. Ramos, Phys. Rev. Lett. 95, 052301 (2005).

[22] D. Jido, J. A. Oller, E. Oset, A. Ramos and U. G. Meissner, Nucl. Phys. A 725, 181 (2003).

[23] C. Garcia-Recio, J. Nieves, E. Ruiz Arriola and M. J. Vicente Vacas, Phys. Rev. D 67, 076009 (2003).

[24] E. Oset, A. Ramos and C. Bennhold, Phys. Lett. B 527, 99 (2002) [Erratum-ibid. B 530, 260 (2002)].

[25] T. Hyodo, S. I. Nam, D. Jido and A. Hosaka, Phys. Rev. C 68, 018201 (2003).

[26] C. Garcia-Recio, M. F. M. Lutz and J. Nieves, Phys. Lett. B 582, 49 (2004).

[27] L. Roca, T. Hyodo and D. Jido, Nucl. Phys. A 809, 65 (2008).

[28] S. Pakvasa and S. F. Tuan, Phys. Lett. B 459, 301 (1999).

[29] P. J. . Fink, G. He, R. H. Landau and J. W. Schnick, Phys. Rev. C 41, 2720 (1990).

[30] T. Hyodo, S. i. Nam, D. Jido and A. Hosaka, Prog. Theor. Phys. 112, 73 (2004).

[31] B. Borasoy, R. Nissler and W. Weise, Eur. Phys. J. A 25, 79 (2005).

[32] J. A. Oller, J. Prades and M. Verbeni, Phys. Rev. Lett. 95, 172502 (2005).

[33] L. Roca, Sourav Sarkar and V. K. Magas, and E. Oset Phys. Rev. C 73, 045208 (2006).

[34] L. Roca, C. Hanhart, E. Oset and U. G. Meissner, Eur. Phys. J. A 27, 373 (2006).

[35] T. Hyodo, A. Hosaka, E. Oset, A. Ramos and M. J. Vicente Vacas, Phys. Rev. C 68, 065203 (2003).

[36] L. S. Geng, E. Oset and M. Doring, Eur. Phys. J. A 32 (2007) 201.

[37] L. S. Geng and E. Oset, Eur. Phys. J. A 34, 405 (2007).

[38] L. S. Geng, E. Oset and B. S. Zou, arXiv:0807.4798 [hep-ph].

[39] J. A. Oller, J. Prades and M. Verbeni, Eur. Phys. J. A 31, 527 (2007).

[40] N. Isgur and G. Karl, Phys. Rev. D 18, 4187 (1978).

[41] S. Capstick and N. Isgur, Phys. Rev. D 34, 2809 (1986).

[42] U. Loring, B. C. Metsch and H. R. Petry, Eur. Phys. J. A 10, 447 (2001).

[43] B. S. Zou, Eur. Phys. J. A 35, 325 (2008).

[44] J. J. Xie, B. S. Zou and H. C. Chiang, Phys. Rev. C 77, 015206 (2008).

[45] A. Sibirtsev, J. Haidenbauer, H. W. Hammer and S. Krewald, Eur. Phys. J. A 27, 269 (2006).

[46] T. Abdullah and F. E. Close, Phys. Rev. D 5, 2332 (1972).

[47] F. E. Close and Z. P. Li, Phys. Rev. D 42, 2194 (1990).

[48] Q. Zhao, J.S. Al-Khalili, and C. Bennhold, Phys. Rev. C 64, 052201(R) (2001).

[49] Q. Zhao, B. Saghai and Z. P. Li, J. Phys. G 28, 1293 (2002).

[50] Q. Zhao, Z. P. Li and C. Bennhold, Phys. Rev. C 58, 2393 (1998); Phys. Lett. B 436, 42 (1998).

[51] J. He, B. Saghai and Z. Li, Phys. Rev. C 78, 035204 (2008).

[52] Z. P. Li, Phys. Rev. D 48, 3070 (1993); 50, 5639 (1994); 52, 4961 (1995); C 52, 1648 (1995);.

[53] Z. P. Li, H. X. Ye and M. H. Lu, Phys. Rev. C 56, 1099 (1997).

[54] Q. Zhao, J. S. Al-Khalili, Z. P. Li and R. L. Workman, Phys. Rev. C 65, 065204 (2002).

[55] X. H. Zhong, Q. Zhao, J. He and B. Saghai, Phys. Rev. C 76, 065205 (2007).

[56] X. H. Zhong and Q. Zhao, Phys. Rev. D 77, 074008 (2008)

[57] X. H. Zhong and Q. Zhao, Phys. Rev. D 78, 014029 (2008).

[58] A. D. Martin, N. M. Queen and G. Violini, Nucl. Phys. B 10, 481 (1969).

[59] P. M. Gensini, R. Hurtado and G. Violini, PiN Newslett. 13, 291 (1997).

[60] A. D. Martin, Nucl. Phys. B 179, 33 (1981).

[61] R. Buttgen, K. Holinde and J. Speth, Phys. Lett. B 163, 305 (1985).

[62] R. Buettgen, K. Holinde, A. Mueller-Groeling, J. Speth and P. Wyborny, Nucl. Phys. A 506, 586 (1990).

[63] A. Mueller- Groeling, K. Holinde and J. Speth, Nucl. Phys. A 513, 557 (1990).

[64] T. Hamaie, M. Arima and K. Masutani, Nucl. Phys. A 591, 675 (1995). 
[65] N. Isgur and G. Karl, Phys. Lett. B72, 109 (1977); Phys. Rev. D 19, 2653 (1979) [Erratum-ibid. 23, 817 (1981)]; 20, 1191 (1979).

[66] Particle Data Group, Y. M. Yao et al., J. Phys. G 33, 1 (2006).

[67] T. V. Brito, F. S. Navarra, M. Nielsen and M. E. Bracco, Phys. Lett. B 608, 69 (2005).

[68] I. Hleiqawi et al. [CLAS Collaboration], Phys. Rev. C 75, 042201(R) (2007).

[69] M. Nanova et al. [CBELSA/TAPS Collaboration], Eur. Phys. J. A 35, 333 (2008).

[70] Q. Zhao and F. E. Close, Phys. Rev. D 74, 094014 (2006).

[71] N. Isgur, G. Karl and R. Koniuk, Phys. Rev. Lett. 41, 1269 (1978) [Erratum-ibid. 45, 1738 (1980)].

[72] A. J. G. Hey, P. J. Litchfield and R. J. Cashmore, Nucl. Phys. B 95, 516 (1975).

[73] M.L. Goldberger and S.B. Treiman, Phys. Rev. 110, 1178 (1958). 